Supporting Information for:

\title{
Discrete Bridging and Terminal Copper Carbenes \\ in Copper-Catalyzed Cyclopropanation
}

Xuliang Dai and Timothy H. Warren*

Georgetown University, Department of Chemistry,

Box 571227, Washington, DC 20057-1227

\section{Contents}

Variable temperature ${ }^{1} \mathrm{H}$ NMR spectra of $\left\{\left[\mathrm{Me}_{2} \mathrm{NN}\right] \mathrm{Cu}\right\}_{2}\left(\mu-\mathrm{CPh}_{2}\right)(3)$ (Figure S1)

Kinetic Plots for Cyclopropanation of Styrene by 8 (Figure S2)

Kinetic Plots for Thermal Decomposition of 8 (Figures S3 - S5)

Fully labeled ORTEP diagrams for compounds 3 and 8 (Figures S6 - S7) with X-ray refinement details and selected bond distances and angles (Tables S1 - S10)

Selected Output for Calculated Structures 6, 7-C $\mathrm{C}_{2}$, and 7- $\mathrm{C}_{2 \mathrm{v}}$ 
Figure S1. Variable temperature ${ }^{1} \mathrm{H} \quad$ NMR spectra $(300 \quad \mathrm{MHz})$ of $\left\{\left[\mathrm{Me}_{2} \mathrm{NN}\right] \mathrm{Cu}\right\}_{2}\left(\mu-\mathrm{CPh}_{2}\right)(3)$ in toluene- $d_{8}$. Full spectrum recorded at $-70{ }^{\circ} \mathrm{C}$ (a, bottom) and variable-temperature inset showing the $\beta$-diketiminato $N$-aryl and backbone methyl groups (b, top).

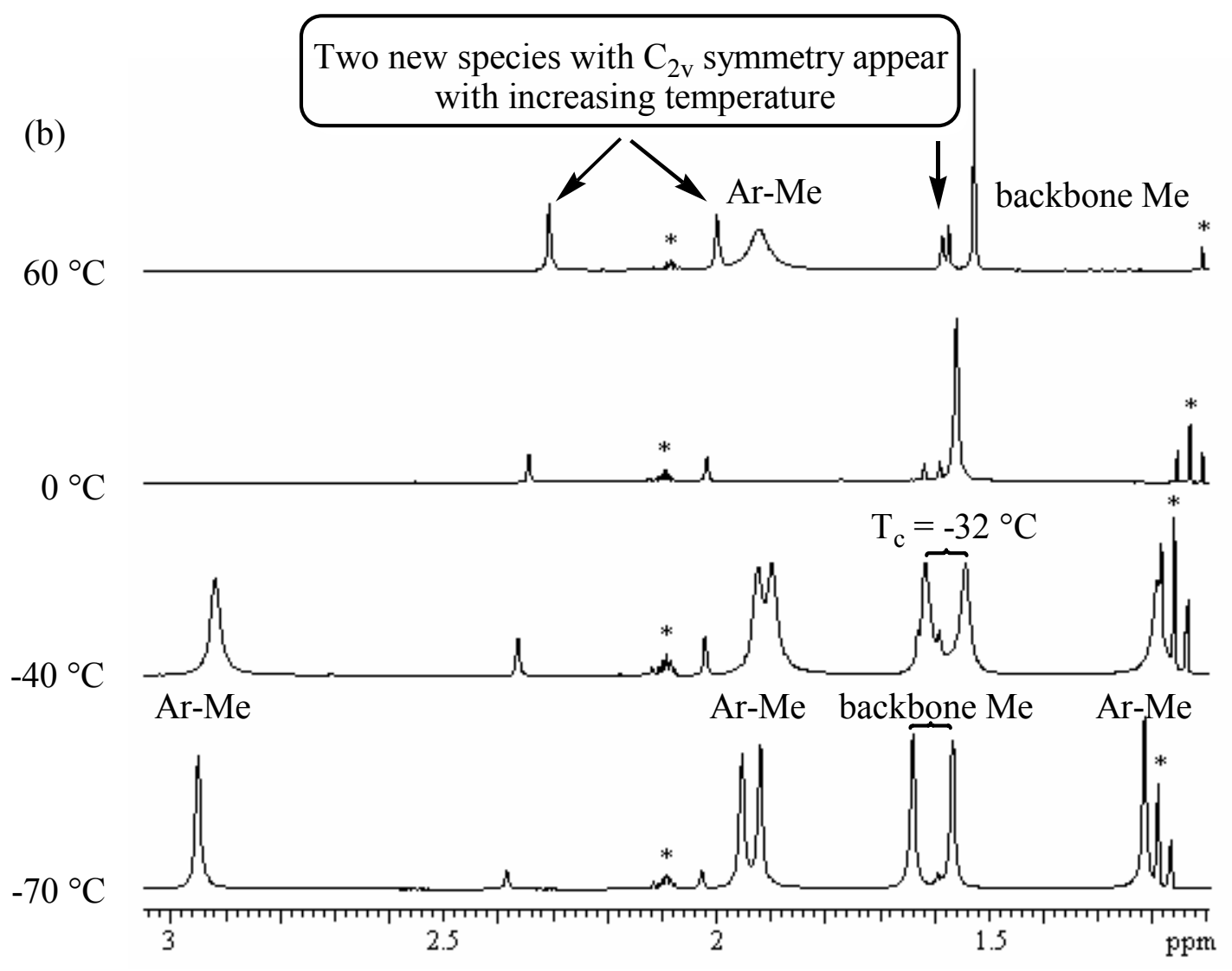

(a)

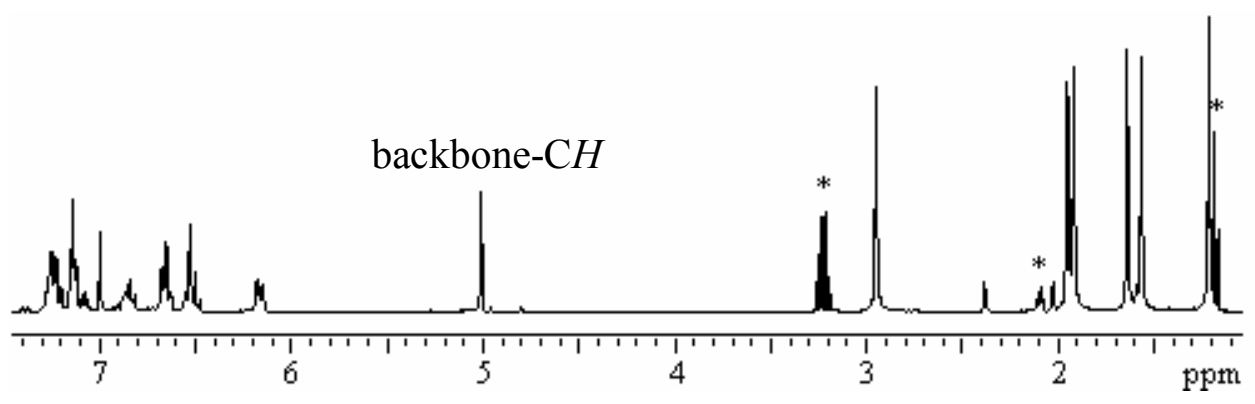


Figure S2. Plots of $\ln \left[\left(\mathrm{A}_{\mathrm{t}}-\mathrm{A}_{\infty}\right) /\left(\mathrm{A}_{0}-\mathrm{A}_{\infty}\right)\right]$ vs. time from $17.5-60.2{ }^{\circ} \mathrm{C}$ for the stoichiometric cyclopropanation of styrene (100 equiv.) by 8 in 1,4-dioxane.

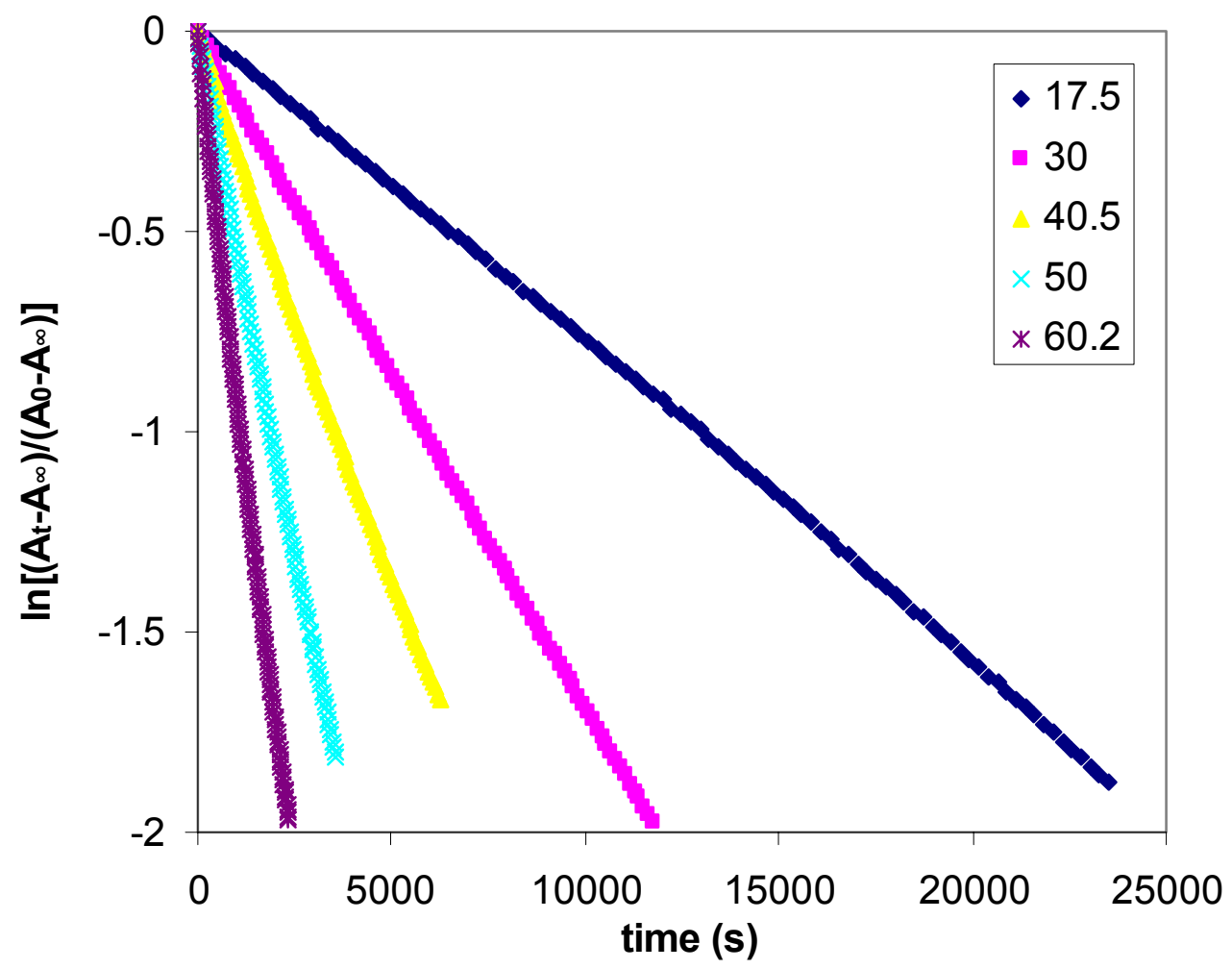

Figure S3. UV-Vis spectra used to monitor the decomposition of $\left[\mathrm{Me}_{3} \mathrm{NN}\right] \mathrm{Cu}=\mathrm{CPh}_{2}(\mathbf{8})$ in dioxane at $32.2^{\circ} \mathrm{C}$.

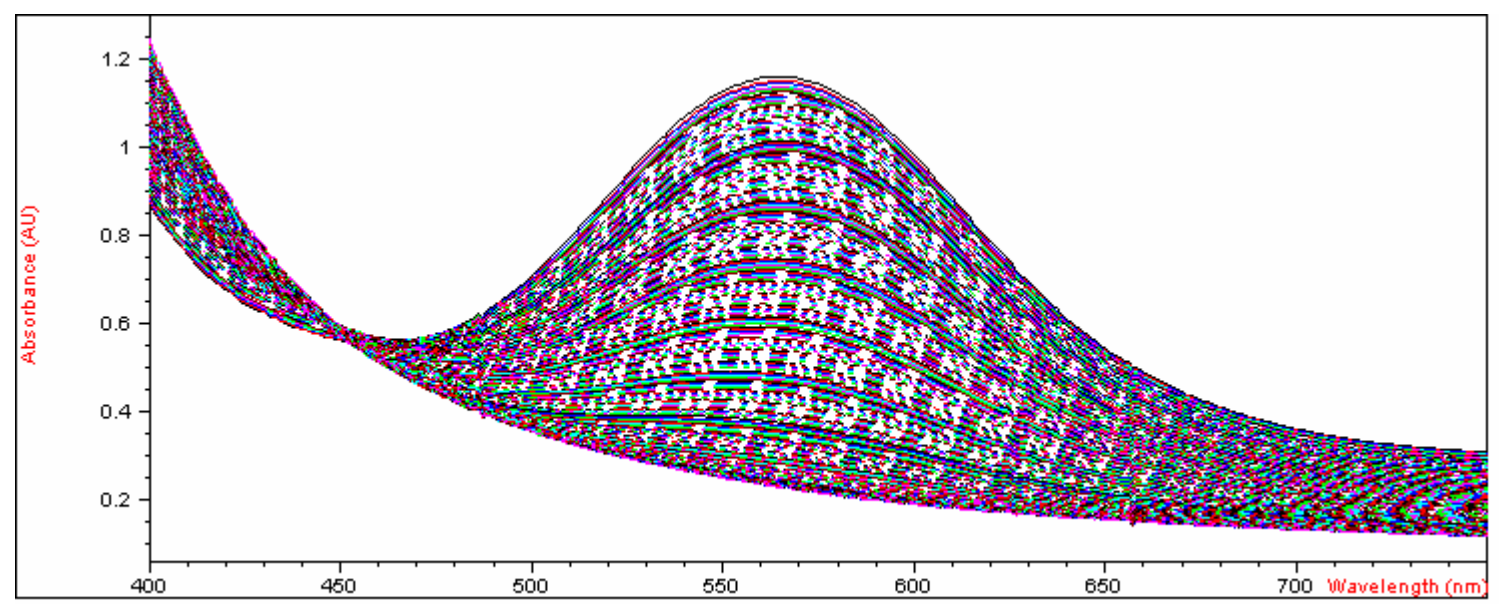


Figure S4. Plots of $\ln \left[\left(\mathrm{A}_{\mathrm{t}}-\mathrm{A}_{\infty}\right) /\left(\mathrm{A}_{0}-\mathrm{A}_{\infty}\right)\right]$ vs. time from $32.2-60.0{ }^{\circ} \mathrm{C}$ for the thermal decomposition of $\left[\mathrm{Me}_{3} \mathrm{NN}\right] \mathrm{Cu}=\mathrm{CPh}_{2}(8)$ in 1,4-dioxane.

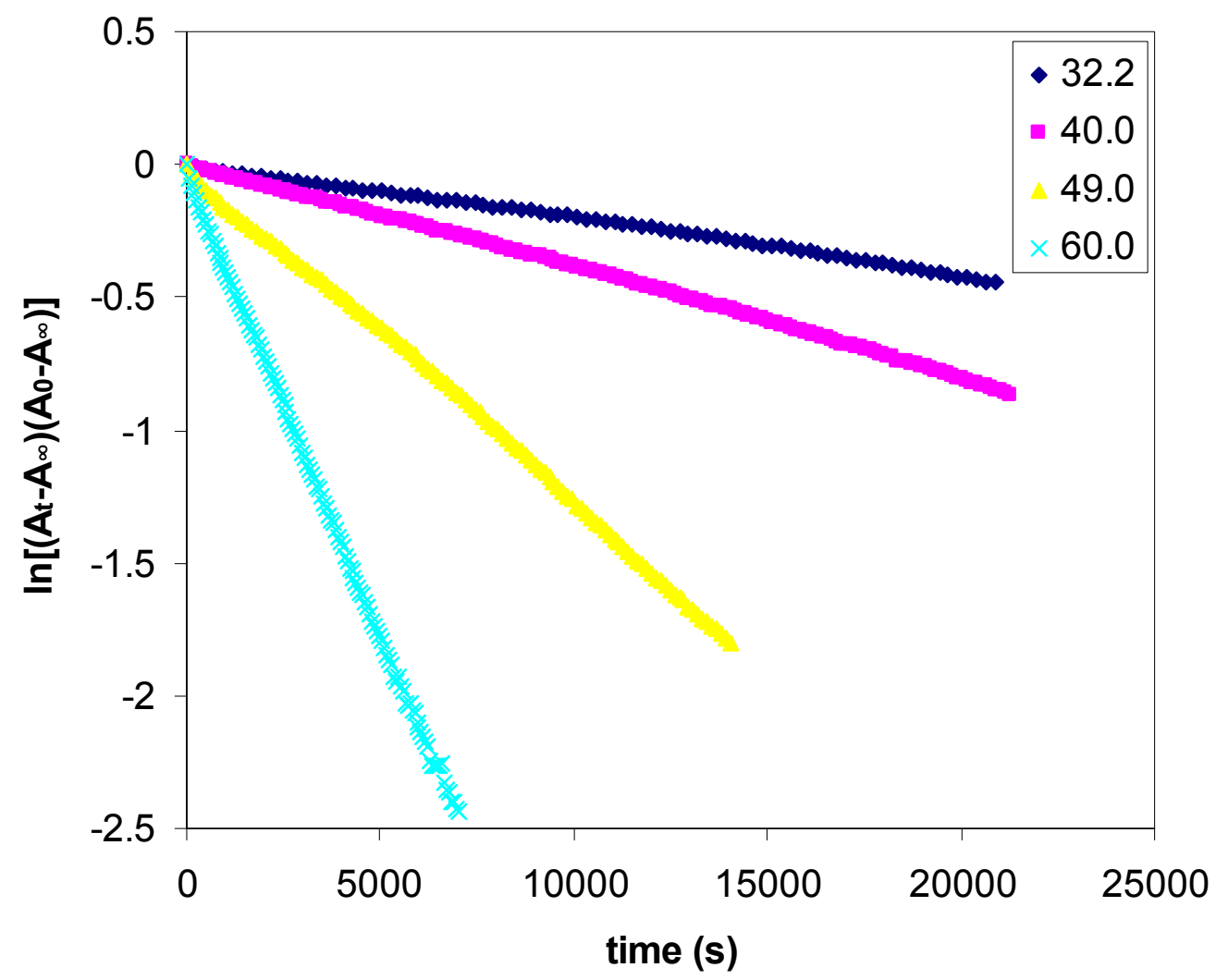

Figure S5. Eyring plot for the decomposition of $\left[\mathrm{Me}_{3} \mathrm{NN}\right] \mathrm{Cu}=\mathrm{CPh}_{2}(\mathbf{8})$ in 1,4-dioxane over the temperature range $32.2-60.0^{\circ} \mathrm{C}$.

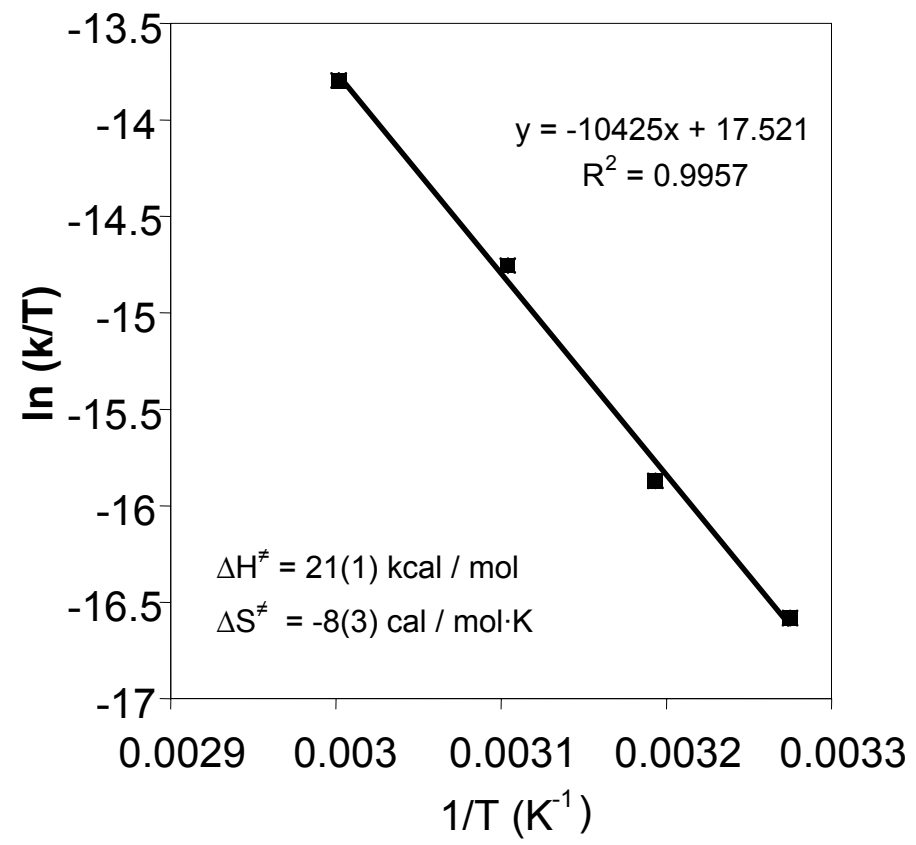


Figure S6. Fully labeled ORTEP diagram of $\left\{\left[\mathrm{Me}_{2} \mathrm{NN}\right] \mathrm{Cu}\right\}_{2}\left(\mu-\mathrm{CPh}_{2}\right)$ (3) (all $\mathrm{H}$ atoms omitted). Selected bond distances $(\AA)$, angles (deg), and twist angles between planes: Cu1-C43 1.922(4), Cu2-C43 1.930(4), Cu1-Cu2 2.4635(7), Cu1-N1 1.965(3), Cu1-N2 1.955(3), Cu2-N3 1.970(3), Cu2-N4 1.978(3), Cu1-C43-Cu2 79.51(14), N1-Cu1-N2 96.11(13), N3-Cu2-N4 95.85(12), C44-C43-C50 115.0(3), N1-Cu1-N2 / C44-C43-C50 89.1, N3-Cu2-N4 / C44-C43-C50 83.1.

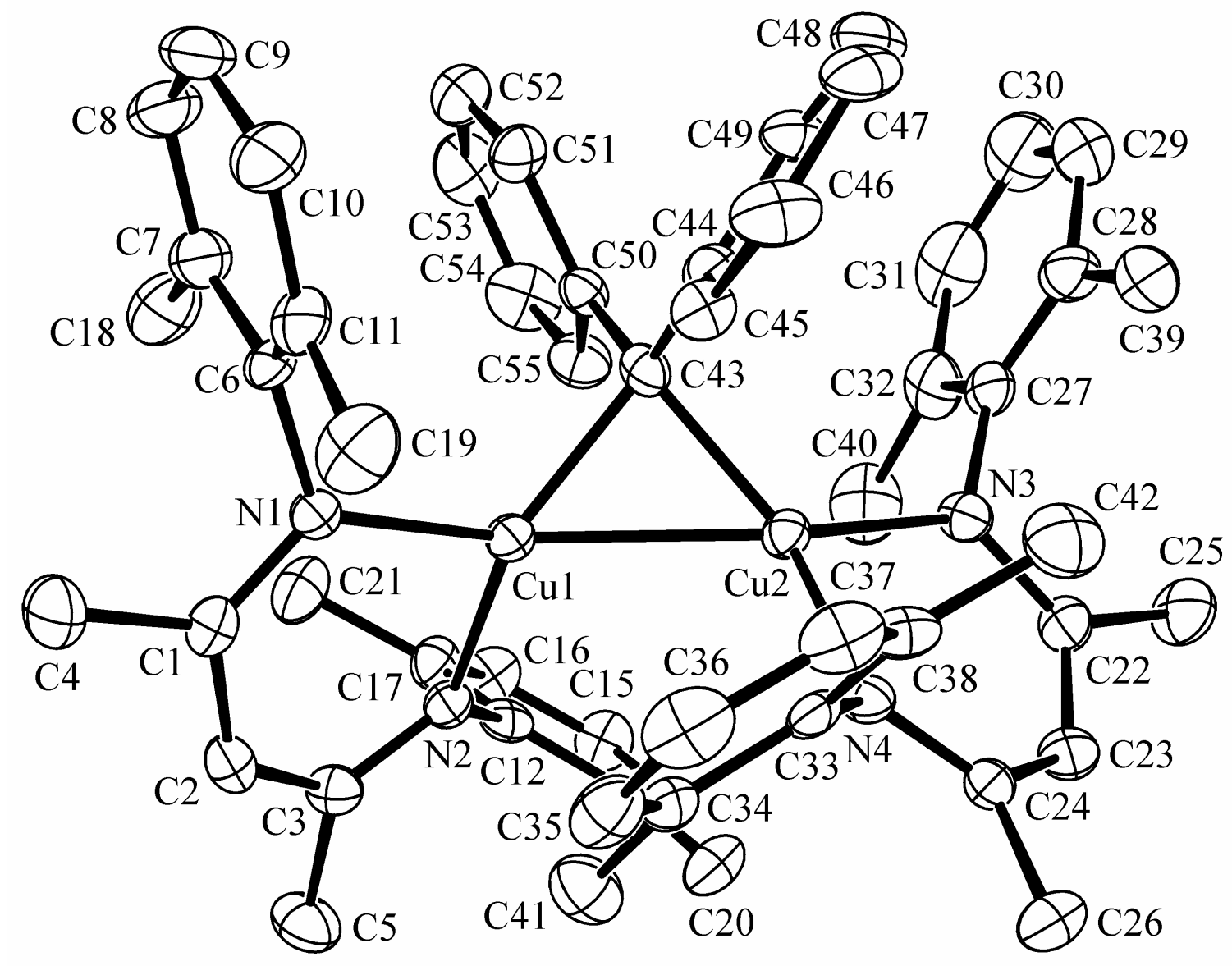


Table S1. Crystal data and structure refinement for $\left\{\left[\mathrm{Me}_{2} \mathrm{NN}\right] \mathrm{Cu}\right\}_{2}\left(\mu-\mathrm{CPh}_{2}\right)(3) \cdot 0.75$ hexane.

Identification code

Empirical formula

Formula weight

Temperature

Wavelength

Crystal system

Space group

Unit cell dimensions

Volume

Z

Density (calculated)

Absorption coefficient

$\mathrm{F}(000)$

Crystal size

Theta range for data collection

Index ranges

Reflections collected / unique

Completeness to theta $=25.00^{\circ}$

Absorption correction

Refinement method

Data / restraints / parameters

Goodness-of-fit on $\mathrm{F}^{2}$

Final R indices [I $>2 \operatorname{sigma}(\mathrm{I})]$

$\mathrm{R}$ indices (all data)

Extinction coefficient

Largest diff. peak and hole
$\left\{\left[\mathrm{Me}_{2} \mathrm{NN}\right] \mathrm{Cu}\right\}_{2}\left(\mu-\mathrm{CPh}_{2}\right)(3)$

$\mathrm{C}_{55} \mathrm{H}_{60} \mathrm{Cu}_{2} \mathrm{~N}_{4}$

904.15

183(2) K

$0.71073 \mathrm{~A}$

Monoclinic

$\mathrm{C} 2 / \mathrm{c}$

$\mathrm{a}=23.906(2) \AA \quad \alpha=90^{\circ}$

$\mathrm{b}=23.681(2) \AA \quad \beta=112.538(2)^{\circ}$

$\mathrm{c}=19.3596(19) \AA \quad \gamma=90^{\circ}$

10122.9(18) $\AA^{3}$

8

$1.187 \mathrm{Mg} / \mathrm{m}^{3}$

$0.878 \mathrm{~mm}^{-1}$

3808

$0.18 \times 0.20 \times 0.50 \mathrm{~mm}$ (dark purple plate)

1.26 to $25.00^{\circ}$.

$-28<=\mathrm{h}<=28,-28<=\mathrm{k}<=28,-23<=\mathrm{l}<=22$

$37875 / 8909[\mathrm{R}($ int $)=0.0857]$

$100.0 \%$

SADABS

Full-matrix least-squares on $\mathrm{F}^{2}$

8909 / 0 / 563

0.900

$\mathrm{R} 1=0.0481, \mathrm{wR} 2=0.1069$

$\mathrm{R} 1=0.0861, \mathrm{wR} 2=0.1166$

none

0.447 and $-0.592 \mathrm{e} \cdot \AA^{-3}$ 
Table S2. Atomic coordinates $\left(\mathrm{x} 10^{4}\right)$ and equivalent isotropic displacement parameters $\left(\AA^{2} \mathrm{x}\right.$ $10^{3}$ ) for $\left\{\left[\mathrm{Me}_{2} \mathrm{NN}\right] \mathrm{Cu}\right\}_{2}\left(\mu-\mathrm{CPh}_{2}\right)(3) \cdot 0.75$ hexane. $\mathrm{U}(\mathrm{eq})$ is defined as one third of the trace of the orthogonalized $\mathrm{U}^{\mathrm{ij}}$ tensor.

\begin{tabular}{|c|c|c|c|c|}
\hline & $\mathrm{x}$ & $\mathrm{y}$ & z & $\mathrm{U}(\mathrm{eq})$ \\
\hline $\mathrm{Cu}(1)$ & $2330(1)$ & $1324(1)$ & $2636(1)$ & $20(1)$ \\
\hline $\mathrm{Cu}(2)$ & $3299(1)$ & $845(1)$ & $3327(1)$ & $20(1)$ \\
\hline $\mathrm{N}(1)$ & $1476(1)$ & $1325(1)$ & $2517(2)$ & $24(1)$ \\
\hline$C(43)$ & $2907(2)$ & $1450(2)$ & $3637(2)$ & $22(1)$ \\
\hline $\mathrm{N}(3)$ & $4182(1)$ & $916(1)$ & $3623(2)$ & $23(1)$ \\
\hline $\mathrm{N}(2)$ & $2174(1)$ & 1401(1) & $1573(2)$ & $20(1)$ \\
\hline $\mathrm{N}(4)$ & $3260(1)$ & $29(1)$ & $3096(2)$ & $19(1)$ \\
\hline $\mathrm{C}(24)$ & $3707(2)$ & $-234(2)$ & $2975(2)$ & $23(1)$ \\
\hline$C(27)$ & $4510(2)$ & $1373(2)$ & $4094(2)$ & $27(1)$ \\
\hline$C(33)$ & $2806(2)$ & $-313(2)$ & $3197(2)$ & $22(1)$ \\
\hline $\mathrm{C}(1)$ & $1036(2)$ & $1219(2)$ & $1867(2)$ & $28(1)$ \\
\hline$C(12)$ & $2603(2)$ & $1688(2)$ & $1332(2)$ & $21(1)$ \\
\hline$C(50)$ & $2924(2)$ & $2074(2)$ & $3616(2)$ & $21(1)$ \\
\hline$C(3)$ & $1640(2)$ & $1265(2)$ & $1049(2)$ & $24(1)$ \\
\hline $\mathrm{C}(38)$ & $2938(2)$ & $-584(2)$ & $3885(2)$ & $25(1)$ \\
\hline$C(13)$ & $3093(2)$ & $1394(2)$ & $1265(2)$ & $20(1)$ \\
\hline$C(23)$ & $4250(2)$ & $23(2)$ & $3046(2)$ & $27(1)$ \\
\hline$C(45)$ & $2695(2)$ & $705(2)$ & $4423(2)$ & $27(1)$ \\
\hline$C(26)$ & $3646(2)$ & $-861(2)$ & $2785(2)$ & $32(1)$ \\
\hline$C(20)$ & $3174(2)$ & $773(2)$ & $1419(2)$ & $33(1)$ \\
\hline$C(51)$ & $2716(2)$ & $2412(2)$ & $4073(2)$ & $28(1)$ \\
\hline$C(6)$ & $1306(2)$ & $1490(2)$ & $3129(2)$ & $25(1)$ \\
\hline$C(37)$ & $2480(2)$ & $-890(2)$ & 3998(2) & $33(1)$ \\
\hline$C(16)$ & $2939(2)$ & $2543(2)$ & $947(2)$ & $32(1)$ \\
\hline$C(34)$ & $2226(2)$ & $-364(2)$ & $2623(2)$ & $24(1)$ \\
\hline$C(14)$ & $3499(2)$ & $1695(2)$ & $1046(2)$ & $29(1)$ \\
\hline$C(22)$ & $4494(2)$ & $533(2)$ & $3411(2)$ & $25(1)$ \\
\hline$C(36)$ & $1910(2)$ & $-933(2)$ & $3446(3)$ & $35(1)$ \\
\hline$C(44)$ & $2987(2)$ & $1204(2)$ & $4376(2)$ & $22(1)$ \\
\hline $\mathrm{C}(11)$ & $1244(2)$ & $1082(2)$ & $3620(2)$ & $28(1)$ \\
\hline
\end{tabular}




\begin{tabular}{|c|c|c|c|c|}
\hline$C(41)$ & $2085(2)$ & $-98(2)$ & $1875(2)$ & $36(1)$ \\
\hline$C(35)$ & $1792(2)$ & $-676(2)$ & $2768(2)$ & $32(1)$ \\
\hline$C(5)$ & $1560(2)$ & $1263(2)$ & $232(2)$ & $39(1)$ \\
\hline$C(2)$ & $1124(2)$ & $1135(2)$ & $1194(2)$ & $30(1)$ \\
\hline$C(46)$ & $2785(2)$ & $453(2)$ & $5098(2)$ & $37(1)$ \\
\hline $\mathrm{C}(48)$ & $3504(2)$ & $1166(2)$ & $5724(2)$ & $35(1)$ \\
\hline$C(55)$ & $3093(2)$ & $2347(2)$ & $3089(2)$ & $31(1)$ \\
\hline C(49) & $3400(2)$ & $1428(2)$ & $5050(2)$ & $27(1)$ \\
\hline $\mathrm{C}(7)$ & $1221(2)$ & $2064(2)$ & $3230(2)$ & $31(1)$ \\
\hline$C(54)$ & $3084(2)$ & $2931(2)$ & $3039(3)$ & $42(1)$ \\
\hline$C(53)$ & $2895(2)$ & $3250(2)$ & $3500(3)$ & $42(1)$ \\
\hline$C(52)$ & $2706(2)$ & 2991(2) & $4011(2)$ & $39(1)$ \\
\hline $\mathrm{C}(9)$ & $1065(2)$ & $1828(2)$ & $4356(2)$ & $43(1)$ \\
\hline$C(10)$ & $1142(2)$ & $1270(2)$ & $4244(2)$ & $40(1)$ \\
\hline$C(47)$ & $3189(2)$ & $683(2)$ & $5751(2)$ & $36(1)$ \\
\hline$C(4)$ & $382(2)$ & $1188(2)$ & 1791(3) & $46(1)$ \\
\hline C(19) & $1238(2)$ & $460(2)$ & $3461(3)$ & $45(1)$ \\
\hline$C(17)$ & $2521(2)$ & $2262(2)$ & $1163(2)$ & $26(1)$ \\
\hline$C(15)$ & $3424(2)$ & $2265(2)$ & $895(2)$ & $33(1)$ \\
\hline$C(21)$ & $1986(2)$ & $2584(2)$ & $1203(2)$ & $37(1)$ \\
\hline$C(42)$ & $3556(2)$ & $-555(2)$ & $4499(2)$ & $39(1)$ \\
\hline$C(28)$ & $4740(2)$ & $1307(2)$ & $4866(2)$ & $33(1)$ \\
\hline$C(32)$ & $4578(2)$ & $1886(2)$ & $3765(3)$ & $37(1)$ \\
\hline$C(25)$ & $5159(2)$ & $632(2)$ & $3585(2)$ & $37(1)$ \\
\hline$C(18)$ & $1242(2)$ & $2494(2)$ & $2675(3)$ & $46(1)$ \\
\hline C(39) & $4690(2)$ & $750(2)$ & $5212(2)$ & $43(1)$ \\
\hline$C(8)$ & $1095(2)$ & $2221(2)$ & $3848(2)$ & $38(1)$ \\
\hline $\mathrm{C}(40)$ & $4383(2)$ & $1946(2)$ & 2939(3) & $53(1)$ \\
\hline$C(29)$ & $5016(2)$ & $1765(2)$ & $5318(3)$ & $49(1)$ \\
\hline$C(30)$ & $5071(2)$ & $2269(2)$ & $5007(3)$ & $58(2)$ \\
\hline $\mathrm{C}(31)$ & $4860(2)$ & $2330(2)$ & $4248(3)$ & $53(1)$ \\
\hline
\end{tabular}


Table S3. Bond lengths $[\AA]$ and angles $\left[^{\circ}\right]$ for $\left\{\left[\mathrm{Me}_{2} \mathrm{NN}\right] \mathrm{Cu}\right\}_{2}\left(\mu-\mathrm{CPh}_{2}\right)(3) \cdot 0.75$ hexane.

\begin{tabular}{|c|c|}
\hline $\mathrm{Cu}(1)-\mathrm{C}(43)$ & $1.922(4)$ \\
\hline $\mathrm{Cu}(1)-\mathrm{N}(2)$ & $1.955(3)$ \\
\hline $\mathrm{Cu}(1)-\mathrm{N}(1)$ & $1.965(3)$ \\
\hline $\mathrm{Cu}(1)-\mathrm{Cu}(2)$ & $2.4635(7)$ \\
\hline $\mathrm{Cu}(2)-\mathrm{C}(43)$ & $1.930(4)$ \\
\hline $\mathrm{Cu}(2)-\mathrm{N}(3)$ & $1.970(3)$ \\
\hline $\mathrm{Cu}(2)-\mathrm{N}(4)$ & $1.978(3)$ \\
\hline $\mathrm{N}(1)-\mathrm{C}(1)$ & $1.318(5)$ \\
\hline$N(1)-C(6)$ & $1.447(4)$ \\
\hline$C(43)-C(50)$ & $1.479(5)$ \\
\hline$C(43)-C(44)$ & $1.490(5)$ \\
\hline $\mathrm{N}(3)-\mathrm{C}(22)$ & $1.334(5)$ \\
\hline $\mathrm{N}(3)-\mathrm{C}(27)$ & $1.439(5)$ \\
\hline $\mathrm{N}(2)-\mathrm{C}(3)$ & $1.331(5)$ \\
\hline $\mathrm{N}(2)-\mathrm{C}(12)$ & $1.448(4)$ \\
\hline $\mathrm{N}(4)-\mathrm{C}(24)$ & $1.333(4)$ \\
\hline $\mathrm{N}(4)-\mathrm{C}(33)$ & $1.426(5)$ \\
\hline $\mathrm{C}(24)-\mathrm{C}(23)$ & $1.393(5)$ \\
\hline$C(24)-C(26)$ & $1.521(5)$ \\
\hline $\mathrm{C}(27)-\mathrm{C}(28)$ & $1.389(5)$ \\
\hline $\mathrm{C}(27)-\mathrm{C}(32)$ & $1.409(6)$ \\
\hline $\mathrm{C}(33)-\mathrm{C}(38)$ & $1.401(5)$ \\
\hline$C(33)-C(34)$ & $1.410(5)$ \\
\hline $\mathrm{C}(1)-\mathrm{C}(2)$ & $1.411(5)$ \\
\hline$C(1)-C(4)$ & $1.514(5)$ \\
\hline$C(12)-C(17)$ & $1.396(5)$ \\
\hline$C(12)-C(13)$ & $1.409(5)$ \\
\hline$C(50)-C(55)$ & $1.392(5)$ \\
\hline$C(50)-C(51)$ & $1.415(5)$ \\
\hline $\mathrm{C}(3)-\mathrm{C}(2)$ & $1.401(5)$ \\
\hline$C(3)-C(5)$ & $1.518(5)$ \\
\hline $\mathrm{C}(38)-\mathrm{C}(37)$ & $1.399(5)$ \\
\hline$C(38)-C(42)$ & $1.503(5)$ \\
\hline$C(13)-C(14)$ & $1.396(5)$ \\
\hline
\end{tabular}




\begin{tabular}{|c|c|}
\hline$C(13)-C(20)$ & $1.499(5)$ \\
\hline$C(23)-C(22)$ & $1.409(5)$ \\
\hline$C(45)-C(46)$ & $1.375(5)$ \\
\hline$C(45)-C(44)$ & $1.393(5)$ \\
\hline$C(51)-C(52)$ & $1.375(5)$ \\
\hline$C(6)-C(11)$ & $1.400(5)$ \\
\hline$C(6)-C(7)$ & $1.400(5)$ \\
\hline$C(37)-C(36)$ & $1.374(6)$ \\
\hline$C(16)-C(15)$ & $1.371(5)$ \\
\hline$C(16)-C(17)$ & $1.391(5)$ \\
\hline$C(34)-C(35)$ & $1.388(5)$ \\
\hline$C(34)-C(41)$ & $1.494(5)$ \\
\hline$C(14)-C(15)$ & $1.378(5)$ \\
\hline$C(22)-C(25)$ & $1.513(5)$ \\
\hline$C(36)-C(35)$ & $1.376(6)$ \\
\hline$C(44)-C(49)$ & $1.404(5)$ \\
\hline$C(11)-C(10)$ & $1.394(5)$ \\
\hline C(11)-C(19) & $1.505(6)$ \\
\hline$C(46)-C(47)$ & $1.377(6)$ \\
\hline C(48)-C(49) & $1.378(5)$ \\
\hline$C(48)-C(47)$ & $1.381(6)$ \\
\hline$C(55)-C(54)$ & $1.386(5)$ \\
\hline$C(7)-C(8)$ & $1.391(5)$ \\
\hline $\mathrm{C}(7)-\mathrm{C}(18)$ & $1.495(6)$ \\
\hline$C(54)-C(53)$ & $1.371(6)$ \\
\hline$C(53)-C(52)$ & $1.379(6)$ \\
\hline C(9)-C(10) & $1.362(6)$ \\
\hline $\mathrm{C}(9)-\mathrm{C}(8)$ & $1.376(6)$ \\
\hline $\mathrm{C}(17)-\mathrm{C}(21)$ & $1.516(5)$ \\
\hline $\mathrm{C}(28)-\mathrm{C}(29)$ & $1.391(6)$ \\
\hline C(28)-C(39) & $1.506(6)$ \\
\hline$C(32)-C(31)$ & $1.396(6)$ \\
\hline$C(32)-C(40)$ & $1.491(6)$ \\
\hline$C(29)-C(30)$ & $1.367(7)$ \\
\hline $\mathrm{C}(30)-\mathrm{C}(31)$ & $1.365(7)$ \\
\hline
\end{tabular}




\begin{tabular}{|c|c|}
\hline $\mathrm{C}(43)-\mathrm{Cu}(1)-\mathrm{N}(2)$ & $145.10(14)$ \\
\hline $\mathrm{C}(43)-\mathrm{Cu}(1)-\mathrm{N}(1)$ & $115.48(14)$ \\
\hline $\mathrm{N}(2)-\mathrm{Cu}(1)-\mathrm{N}(1)$ & $96.11(13)$ \\
\hline $\mathrm{C}(43)-\mathrm{Cu}(1)-\mathrm{Cu}(2)$ & $50.40(11)$ \\
\hline $\mathrm{N}(2)-\mathrm{Cu}(1)-\mathrm{Cu}(2)$ & $111.95(9)$ \\
\hline $\mathrm{N}(1)-\mathrm{Cu}(1)-\mathrm{Cu}(2)$ & $141.54(9)$ \\
\hline $\mathrm{C}(43)-\mathrm{Cu}(2)-\mathrm{N}(3)$ & $116.13(14)$ \\
\hline $\mathrm{C}(43)-\mathrm{Cu}(2)-\mathrm{N}(4)$ & $143.99(14)$ \\
\hline $\mathrm{N}(3)-\mathrm{Cu}(2)-\mathrm{N}(4)$ & $95.85(12)$ \\
\hline $\mathrm{C}(43)-\mathrm{Cu}(2)-\mathrm{Cu}(1)$ & $50.09(11)$ \\
\hline $\mathrm{N}(3)-\mathrm{Cu}(2)-\mathrm{Cu}(1)$ & $143.01(9)$ \\
\hline $\mathrm{N}(4)-\mathrm{Cu}(2)-\mathrm{Cu}(1)$ & $111.95(9)$ \\
\hline $\mathrm{C}(1)-\mathrm{N}(1)-\mathrm{C}(6)$ & $117.5(3)$ \\
\hline $\mathrm{C}(1)-\mathrm{N}(1)-\mathrm{Cu}(1)$ & $121.6(3)$ \\
\hline $\mathrm{C}(6)-\mathrm{N}(1)-\mathrm{Cu}(1)$ & $120.6(2)$ \\
\hline$C(50)-C(43)-C(44)$ & $115.0(3)$ \\
\hline $\mathrm{C}(50)-\mathrm{C}(43)-\mathrm{Cu}(1)$ & $98.3(2)$ \\
\hline $\mathrm{C}(44)-\mathrm{C}(43)-\mathrm{Cu}(1)$ & $133.3(3)$ \\
\hline $\mathrm{C}(50)-\mathrm{C}(43)-\mathrm{Cu}(2)$ & $135.4(3)$ \\
\hline $\mathrm{C}(44)-\mathrm{C}(43)-\mathrm{Cu}(2)$ & $96.2(2)$ \\
\hline $\mathrm{Cu}(1)-\mathrm{C}(43)-\mathrm{Cu}(2)$ & $79.51(14)$ \\
\hline $\mathrm{C}(22)-\mathrm{N}(3)-\mathrm{C}(27)$ & $118.2(3)$ \\
\hline $\mathrm{C}(22)-\mathrm{N}(3)-\mathrm{Cu}(2)$ & $121.0(3)$ \\
\hline $\mathrm{C}(27)-\mathrm{N}(3)-\mathrm{Cu}(2)$ & $120.8(2)$ \\
\hline $\mathrm{C}(3)-\mathrm{N}(2)-\mathrm{C}(12)$ & $117.3(3)$ \\
\hline $\mathrm{C}(3)-\mathrm{N}(2)-\mathrm{Cu}(1)$ & $121.3(2)$ \\
\hline $\mathrm{C}(12)-\mathrm{N}(2)-\mathrm{Cu}(1)$ & $120.7(2)$ \\
\hline $\mathrm{C}(24)-\mathrm{N}(4)-\mathrm{C}(33)$ & $117.4(3)$ \\
\hline $\mathrm{C}(24)-\mathrm{N}(4)-\mathrm{Cu}(2)$ & $121.6(3)$ \\
\hline $\mathrm{C}(33)-\mathrm{N}(4)-\mathrm{Cu}(2)$ & $119.8(2)$ \\
\hline $\mathrm{N}(4)-\mathrm{C}(24)-\mathrm{C}(23)$ & $124.0(4)$ \\
\hline $\mathrm{N}(4)-\mathrm{C}(24)-\mathrm{C}(26)$ & $119.3(3)$ \\
\hline$C(23)-C(24)-C(26)$ & $116.7(3)$ \\
\hline $\mathrm{C}(28)-\mathrm{C}(27)-\mathrm{C}(32)$ & $121.1(4)$ \\
\hline $\mathrm{C}(28)-\mathrm{C}(27)-\mathrm{N}(3)$ & $119.4(4)$ \\
\hline $\mathrm{C}(32)-\mathrm{C}(27)-\mathrm{N}(3)$ & $119.5(4)$ \\
\hline
\end{tabular}




$\begin{array}{ll}\mathrm{C}(38)-\mathrm{C}(33)-\mathrm{C}(34) & 120.3(4) \\ \mathrm{C}(38)-\mathrm{C}(33)-\mathrm{N}(4) & 119.1(3) \\ \mathrm{C}(34)-\mathrm{C}(33)-\mathrm{N}(4) & 120.6(3) \\ \mathrm{N}(1)-\mathrm{C}(1)-\mathrm{C}(2) & 124.2(4) \\ \mathrm{N}(1)-\mathrm{C}(1)-\mathrm{C}(4) & 121.2(4) \\ \mathrm{C}(2)-\mathrm{C}(1)-\mathrm{C}(4) & 114.6(4) \\ \mathrm{C}(17)-\mathrm{C}(12)-\mathrm{C}(13) & 120.4(3) \\ \mathrm{C}(17)-\mathrm{C}(12)-\mathrm{N}(2) & 118.6(3) \\ \mathrm{C}(13)-\mathrm{C}(12)-\mathrm{N}(2) & 120.9(3) \\ \mathrm{C}(55)-\mathrm{C}(50)-\mathrm{C}(51) & 117.8(4) \\ \mathrm{C}(55)-\mathrm{C}(50)-\mathrm{C}(43) & 120.0(3) \\ \mathrm{C}(51)-\mathrm{C}(50)-\mathrm{C}(43) & 121.9(3) \\ \mathrm{N}(2)-\mathrm{C}(3)-\mathrm{C}(2) & 124.2(3) \\ \mathrm{N}(2)-\mathrm{C}(3)-\mathrm{C}(5) & 119.7(3) \\ \mathrm{C}(2)-\mathrm{C}(3)-\mathrm{C}(5) & 116.0(3) \\ \mathrm{C}(37)-\mathrm{C}(38)-\mathrm{C}(33) & 118.8(4) \\ \mathrm{C}(37)-\mathrm{C}(38)-\mathrm{C}(42) & 119.5(4) \\ \mathrm{C}(33)-\mathrm{C}(38)-\mathrm{C}(42) & 121.7(4) \\ \mathrm{C}(14)-\mathrm{C}(13)-\mathrm{C}(12) & 118.5(3) \\ \mathrm{C}(14)-\mathrm{C}(13)-\mathrm{C}(20) & 120.6(3) \\ \mathrm{C}(12)-\mathrm{C}(13)-\mathrm{C}(20) & 120.9(3) \\ \mathrm{C}(24)-\mathrm{C}(23)-\mathrm{C}(22) & 127.9(4) \\ \mathrm{C}(46)-\mathrm{C}(45)-\mathrm{C}(44) & 122.0(4) \\ \mathrm{C}(52)-\mathrm{C}(51)-\mathrm{C}(50) & 120.5(4) \\ \mathrm{C}(11)-\mathrm{C}(6)-\mathrm{C}(7) & 121.2(4) \\ \mathrm{C}(11)-\mathrm{C}(6)-\mathrm{N}(1) & 120.3(3) \\ \mathrm{C}(7)-\mathrm{C}(6)-\mathrm{N}(1) & 118.5(4) \\ \mathrm{C}(36)-\mathrm{C}(37)-\mathrm{C}(38) & 121.2(4) \\ \mathrm{C}(15)-\mathrm{C}(16)-\mathrm{C}(17) & 121.3(4) \\ \mathrm{C}(35)-\mathrm{C}(34)-\mathrm{C}(33) & 118.2(4) \\ \mathrm{C}(35)-\mathrm{C}(34)-\mathrm{C}(41) & 120.7(4) \\ \mathrm{C}(33)-\mathrm{C}(34)-\mathrm{C}(41) & 121.1(4) \\ \mathrm{C}(15)-\mathrm{C}(14)-\mathrm{C}(13) & 121.0(4) \\ \mathrm{N}(3)-\mathrm{C}(22)-\mathrm{C}(23) & 124.4(4) \\ \mathrm{N}(3)-\mathrm{C}(22)-\mathrm{C}(25) & 119.4(4) \\ \mathrm{C}(23)-\mathrm{C}(22)-\mathrm{C}(25) & 116.2(3)\end{array}$




$\begin{array}{ll}\mathrm{C}(37)-\mathrm{C}(36)-\mathrm{C}(35) & 119.3(4) \\ \mathrm{C}(45)-\mathrm{C}(44)-\mathrm{C}(49) & 116.9(4) \\ \mathrm{C}(45)-\mathrm{C}(44)-\mathrm{C}(43) & 120.8(3) \\ \mathrm{C}(49)-\mathrm{C}(44)-\mathrm{C}(43) & 122.0(3) \\ \mathrm{C}(10)-\mathrm{C}(11)-\mathrm{C}(6) & 117.8(4) \\ \mathrm{C}(10)-\mathrm{C}(11)-\mathrm{C}(19) & 119.8(4) \\ \mathrm{C}(6)-\mathrm{C}(11)-\mathrm{C}(19) & 122.2(4) \\ \mathrm{C}(36)-\mathrm{C}(35)-\mathrm{C}(34) & 122.2(4) \\ \mathrm{C}(3)-\mathrm{C}(2)-\mathrm{C}(1) & 127.4(4) \\ \mathrm{C}(45)-\mathrm{C}(46)-\mathrm{C}(47) & 120.0(4) \\ \mathrm{C}(49)-\mathrm{C}(48)-\mathrm{C}(47) & 120.3(4) \\ \mathrm{C}(54)-\mathrm{C}(55)-\mathrm{C}(50) & 120.8(4) \\ \mathrm{C}(48)-\mathrm{C}(49)-\mathrm{C}(44) & 121.1(4) \\ \mathrm{C}(8)-\mathrm{C}(7)-\mathrm{C}(6) & 118.0(4) \\ \mathrm{C}(8)-\mathrm{C}(7)-\mathrm{C}(18) & 121.0(4) \\ \mathrm{C}(6)-\mathrm{C}(7)-\mathrm{C}(18) & 121.0(4) \\ \mathrm{C}(53)-\mathrm{C}(54)-\mathrm{C}(55) & 120.3(4) \\ \mathrm{C}(54)-\mathrm{C}(53)-\mathrm{C}(52) & 120.0(4) \\ \mathrm{C}(51)-\mathrm{C}(52)-\mathrm{C}(53) & 120.5(4) \\ \mathrm{C}(10)-\mathrm{C}(9)-\mathrm{C}(8) & 119.6(4) \\ \mathrm{C}(9)-\mathrm{C}(10)-\mathrm{C}(11) & 121.8(4) \\ \mathrm{C}(46)-\mathrm{C}(47)-\mathrm{C}(48) & 119.6(4) \\ \mathrm{C}(16)-\mathrm{C}(17)-\mathrm{C}(12) & 118.9(4) \\ \mathrm{C}(16)-\mathrm{C}(17)-\mathrm{C}(21) & 119.5(4) \\ \mathrm{C}(12)-\mathrm{C}(17)-\mathrm{C}(21) & 121.6(4) \\ \mathrm{C}(16)-\mathrm{C}(15)-\mathrm{C}(14) & 120.0(4) \\ \mathrm{C}(27)-\mathrm{C}(28)-\mathrm{C}(29) & 119.1(4) \\ \mathrm{C}(27)-\mathrm{C}(28)-\mathrm{C}(39) & 120.8(4) \\ \mathrm{C}(29)-\mathrm{C}(28)-\mathrm{C}(39) & 120.1(4) \\ \mathrm{C}(31)-\mathrm{C}(32)-\mathrm{C}(27) & 117.1(4) \\ \mathrm{C}(31)-\mathrm{C}(32)-\mathrm{C}(40) & 121.3(4) \\ \mathrm{C}(27)-\mathrm{C}(32)-\mathrm{C}(40) & 121.5(4) \\ \mathrm{C}(9)-\mathrm{C}(8)-\mathrm{C}(7) & 121.5(4) \\ & \end{array}$


Table S4. Anisotropic displacement parameters $\left(\AA^{2} \times 10^{2}\right)$ for $\left\{\left[\mathrm{Me}_{2} \mathrm{NN}\right] \mathrm{Cu}\right\}_{2}\left(\mu-\mathrm{CPh}_{2}\right)$ (3) $\cdot 0.75$ hexane. The anisotropic displacement factor exponent takes the form: $-2 \pi^{2}\left[\mathrm{~h}^{2}\right.$ $\mathrm{a}^{*^{2}} \mathrm{U}^{11}+\ldots+2 \mathrm{~h} \mathrm{k} \mathrm{a*} \mathrm{b}^{*} \mathrm{U}^{12}$ ]

\begin{tabular}{|c|c|c|c|c|c|c|}
\hline & $\mathrm{U}^{11}$ & $\mathrm{U}^{22}$ & $\mathrm{U}^{33}$ & $\mathrm{U}^{23}$ & $\mathrm{U}^{13}$ & $\mathrm{U}^{12}$ \\
\hline $\mathrm{Cu}(1)$ & $19(1)$ & $23(1)$ & $19(1)$ & $-1(1)$ & $9(1)$ & $0(1)$ \\
\hline $\mathrm{Cu}(2)$ & $18(1)$ & $21(1)$ & $21(1)$ & $-1(1)$ & $9(1)$ & $0(1)$ \\
\hline $\mathrm{N}(1)$ & $19(2)$ & $33(2)$ & $20(2)$ & $-5(2)$ & $9(2)$ & $0(2)$ \\
\hline$C(43)$ & $17(2)$ & $28(2)$ & $20(2)$ & $1(2)$ & $7(2)$ & $-2(2)$ \\
\hline $\mathrm{N}(3)$ & $22(2)$ & $27(2)$ & $22(2)$ & $-3(2)$ & $10(2)$ & $-2(2)$ \\
\hline $\mathrm{N}(2)$ & $22(2)$ & $23(2)$ & $20(2)$ & $1(1)$ & $12(2)$ & $2(2)$ \\
\hline $\mathrm{N}(4)$ & $21(2)$ & $22(2)$ & $17(2)$ & $-2(1)$ & $9(2)$ & $-2(2)$ \\
\hline$C(24)$ & $28(2)$ & $24(2)$ & $15(2)$ & $4(2)$ & $7(2)$ & $7(2)$ \\
\hline $\mathrm{C}(27)$ & $14(2)$ & $32(2)$ & $34(3)$ & $-7(2)$ & $9(2)$ & $-2(2)$ \\
\hline$C(33)$ & $26(2)$ & $16(2)$ & $26(2)$ & $-4(2)$ & $14(2)$ & $6(2)$ \\
\hline $\mathrm{C}(1)$ & $23(2)$ & $32(2)$ & $31(2)$ & $-1(2)$ & $11(2)$ & $5(2)$ \\
\hline$C(12)$ & $28(2)$ & $22(2)$ & $11(2)$ & $3(2)$ & $6(2)$ & $0(2)$ \\
\hline$C(50)$ & $20(2)$ & $21(2)$ & $18(2)$ & $-2(2)$ & $4(2)$ & $-3(2)$ \\
\hline$C(3)$ & $24(2)$ & $26(2)$ & $21(2)$ & $-2(2)$ & $9(2)$ & $0(2)$ \\
\hline$C(38)$ & $39(3)$ & $16(2)$ & $23(2)$ & $-2(2)$ & $17(2)$ & $-3(2)$ \\
\hline$C(13)$ & $22(2)$ & $22(2)$ & $22(2)$ & $-2(2)$ & $13(2)$ & $4(2)$ \\
\hline $\mathrm{C}(23)$ & $28(2)$ & $37(3)$ & $19(2)$ & $-1(2)$ & $14(2)$ & $3(2)$ \\
\hline$C(45)$ & $31(2)$ & $25(2)$ & $27(2)$ & $0(2)$ & $14(2)$ & $0(2)$ \\
\hline$C(26)$ & $40(3)$ & $26(2)$ & $35(3)$ & $-3(2)$ & $21(2)$ & $4(2)$ \\
\hline$C(20)$ & $41(3)$ & $28(2)$ & $36(3)$ & $4(2)$ & $24(2)$ & $7(2)$ \\
\hline $\mathrm{C}(51)$ & $32(3)$ & $22(2)$ & $30(2)$ & $2(2)$ & $12(2)$ & $5(2)$ \\
\hline$C(6)$ & $15(2)$ & $35(3)$ & $26(2)$ & $-11(2)$ & $11(2)$ & $-2(2)$ \\
\hline$C(37)$ & $44(3)$ & $25(2)$ & $38(3)$ & $-1(2)$ & $26(2)$ & $-4(2)$ \\
\hline$C(16)$ & $43(3)$ & $22(2)$ & $36(3)$ & $10(2)$ & $22(2)$ & $1(2)$ \\
\hline$C(34)$ & $24(2)$ & $21(2)$ & $26(2)$ & $-5(2)$ & $8(2)$ & $2(2)$ \\
\hline$C(14)$ & $26(2)$ & $36(3)$ & $27(2)$ & $2(2)$ & $11(2)$ & $4(2)$ \\
\hline$C(22)$ & $20(2)$ & $35(3)$ & $24(2)$ & $2(2)$ & $12(2)$ & $2(2)$ \\
\hline$C(36)$ & $42(3)$ & $24(2)$ & $51(3)$ & $-4(2)$ & $31(3)$ & $-12(2)$ \\
\hline$C(44)$ & $25(2)$ & $20(2)$ & $25(2)$ & $2(2)$ & $12(2)$ & $6(2)$ \\
\hline
\end{tabular}




\begin{tabular}{|c|c|c|c|c|c|c|}
\hline $\mathrm{C}(11)$ & $20(2)$ & $36(2)$ & $34(3)$ & $-3(2)$ & $17(2)$ & $-4(2)$ \\
\hline$C(41)$ & $39(3)$ & $29(2)$ & $36(3)$ & $0(2)$ & $10(2)$ & $-6(2)$ \\
\hline$C(35)$ & $30(3)$ & $24(2)$ & $47(3)$ & $-5(2)$ & $21(2)$ & $-3(2)$ \\
\hline$C(5)$ & $35(3)$ & $59(3)$ & $21(2)$ & $-5(2)$ & $11(2)$ & $-6(2)$ \\
\hline$C(2)$ & $24(2)$ & $41(3)$ & $22(2)$ & $-4(2)$ & $5(2)$ & $1(2)$ \\
\hline$C(46)$ & $60(3)$ & $26(2)$ & $34(3)$ & $2(2)$ & $29(3)$ & $-4(2)$ \\
\hline$C(48)$ & $51(3)$ & $28(3)$ & $24(2)$ & $-2(2)$ & $12(2)$ & $5(2)$ \\
\hline$C(55)$ & $35(3)$ & $32(3)$ & $26(2)$ & $-1(2)$ & $14(2)$ & $-5(2)$ \\
\hline$C(49)$ & $33(3)$ & $22(2)$ & $25(2)$ & $-5(2)$ & $10(2)$ & $-2(2)$ \\
\hline$C(7)$ & $28(3)$ & $35(3)$ & $32(3)$ & $-7(2)$ & $13(2)$ & $-3(2)$ \\
\hline $\mathrm{C}(54)$ & $57(3)$ & $32(3)$ & $37(3)$ & $9(2)$ & $19(3)$ & $-9(2)$ \\
\hline$C(53)$ & $52(3)$ & $20(2)$ & $45(3)$ & $9(2)$ & 11(3) & $3(2)$ \\
\hline$C(52)$ & $40(3)$ & $33(3)$ & $41(3)$ & $-5(2)$ & $11(2)$ & $10(2)$ \\
\hline$C(9)$ & $31(3)$ & $70(4)$ & $28(3)$ & $-20(3)$ & $13(2)$ & $-6(3)$ \\
\hline$C(10)$ & $34(3)$ & $56(3)$ & $37(3)$ & $-5(2)$ & $20(2)$ & $-2(2)$ \\
\hline$C(47)$ & $62(3)$ & $29(3)$ & $20(2)$ & $6(2)$ & $21(2)$ & $13(2)$ \\
\hline$C(4)$ & $25(3)$ & $71(4)$ & $42(3)$ & $-12(3)$ & $13(2)$ & $-2(2)$ \\
\hline$C(19)$ & $40(3)$ & $39(3)$ & $61(3)$ & $-1(3)$ & $27(3)$ & $-5(2)$ \\
\hline$C(17)$ & $28(2)$ & $29(2)$ & $23(2)$ & $6(2)$ & $12(2)$ & $7(2)$ \\
\hline$C(15)$ & $34(3)$ & $33(3)$ & $37(3)$ & $5(2)$ & $19(2)$ & $-3(2)$ \\
\hline$C(21)$ & $42(3)$ & $37(3)$ & $40(3)$ & $10(2)$ & $23(2)$ & $13(2)$ \\
\hline$C(42)$ & $47(3)$ & $43(3)$ & $29(3)$ & $3(2)$ & $17(2)$ & $-1(2)$ \\
\hline$C(28)$ & $27(3)$ & $34(3)$ & $35(3)$ & $-10(2)$ & $9(2)$ & $-2(2)$ \\
\hline$C(32)$ & $23(3)$ & $40(3)$ & $47(3)$ & $0(2)$ & $11(2)$ & $-6(2)$ \\
\hline$C(25)$ & $28(3)$ & $44(3)$ & $44(3)$ & $-6(2)$ & $21(2)$ & $-2(2)$ \\
\hline$C(18)$ & $52(3)$ & $32(3)$ & $57(3)$ & $-2(2)$ & $24(3)$ & $8(2)$ \\
\hline$C(39)$ & $49(3)$ & $47(3)$ & $29(3)$ & $3(2)$ & $9(2)$ & $13(2)$ \\
\hline$C(8)$ & $38(3)$ & $41(3)$ & $40(3)$ & $-17(2)$ & $20(2)$ & $-5(2)$ \\
\hline$C(40)$ & $52(3)$ & $64(4)$ & $53(3)$ & $12(3)$ & $30(3)$ & $0(3)$ \\
\hline$C(29)$ & $39(3)$ & $56(4)$ & $40(3)$ & $-11(3)$ & $3(2)$ & $9(3)$ \\
\hline$C(30)$ & $39(3)$ & $48(3)$ & $76(4)$ & $-24(3)$ & $9(3)$ & $-10(3)$ \\
\hline $\mathrm{C}(31)$ & $40(3)$ & $38(3)$ & $82(4)$ & $-4(3)$ & $22(3)$ & $-6(3)$ \\
\hline
\end{tabular}


Table S5. Hydrogen coordinates ( x 10^4) and isotropic displacement parameters $\left(\AA^{2} \mathrm{x}\right.$ $\left.10^{2}\right)$ for $\left\{\left[\mathrm{Me}_{2} \mathrm{NN}\right] \mathrm{Cu}\right\}_{2}\left(\mu-\mathrm{CPh}_{2}\right)(3) \cdot 0.75$ hexane.

\begin{tabular}{|c|c|c|c|c|}
\hline & $\mathrm{x}$ & $\mathrm{y}$ & z & $\mathrm{U}(\mathrm{eq})$ \\
\hline $\mathrm{H}(23)$ & 4486 & -170 & 2821 & 32 \\
\hline $\mathrm{H}(45)$ & 2425 & 533 & 3976 & 32 \\
\hline $\mathrm{H}(26 \mathrm{~A})$ & 3732 & -1081 & 3243 & 48 \\
\hline $\mathrm{H}(26 \mathrm{~B})$ & 3934 & -964 & 2557 & 48 \\
\hline $\mathrm{H}(26 \mathrm{C})$ & 3232 & -940 & 2433 & 48 \\
\hline $\mathrm{H}(20 \mathrm{~A})$ & 3531 & 641 & 1335 & 49 \\
\hline $\mathrm{H}(20 \mathrm{~B})$ & 2814 & 571 & 1085 & 49 \\
\hline $\mathrm{H}(20 \mathrm{C})$ & 3229 & 701 & 1940 & 49 \\
\hline $\mathrm{H}(51)$ & 2584 & 2238 & 4426 & 33 \\
\hline $\mathrm{H}(37)$ & 2564 & -1072 & 4464 & 39 \\
\hline $\mathrm{H}(16)$ & 2887 & 2934 & 833 & 38 \\
\hline $\mathrm{H}(14)$ & 3833 & 1504 & 1000 & 35 \\
\hline H(36) & 1602 & -1138 & 3533 & 42 \\
\hline $\mathrm{H}(41 \mathrm{~A})$ & 1664 & -181 & 1550 & 54 \\
\hline $\mathrm{H}(41 \mathrm{~B})$ & 2357 & -250 & 1650 & 54 \\
\hline $\mathrm{H}(41 \mathrm{C})$ & 2140 & 312 & 1935 & 54 \\
\hline $\mathrm{H}(35)$ & 1399 & -712 & 2386 & 38 \\
\hline $\mathrm{H}(5 \mathrm{~A})$ & 1559 & 1652 & 60 & 58 \\
\hline $\mathrm{H}(5 \mathrm{~B})$ & 1175 & 1081 & -68 & 58 \\
\hline $\mathrm{H}(5 \mathrm{C})$ & 1894 & 1054 & 177 & 58 \\
\hline $\mathrm{H}(2)$ & 795 & 971 & 793 & 36 \\
\hline $\mathrm{H}(46)$ & 2569 & 119 & 5112 & 44 \\
\hline $\mathrm{H}(48)$ & 3794 & 1318 & 6172 & 42 \\
\hline $\mathrm{H}(55)$ & 3216 & 2129 & 2759 & 37 \\
\hline H(49) & 3610 & 1766 & 5041 & 32 \\
\hline $\mathrm{H}(54)$ & 3210 & 3110 & 2685 & 50 \\
\hline $\mathrm{H}(53)$ & 2895 & 3650 & 3467 & 50 \\
\hline $\mathrm{H}(52)$ & 2567 & 3214 & 4321 & 47 \\
\hline $\mathrm{H}(9)$ & 992 & 1945 & 4783 & 51 \\
\hline
\end{tabular}




\begin{tabular}{|c|c|c|c|c|}
\hline $\mathrm{H}(10)$ & 1125 & 1002 & 4600 & 48 \\
\hline $\mathrm{H}(47)$ & 3251 & 511 & 6218 & 43 \\
\hline $\mathrm{H}(4 \mathrm{~A})$ & 345 & 913 & 2149 & 69 \\
\hline $\mathrm{H}(4 \mathrm{~B})$ & 127 & 1071 & 1282 & 69 \\
\hline $\mathrm{H}(4 \mathrm{C})$ & 252 & 1560 & 1893 & 69 \\
\hline $\mathrm{H}(19 \mathrm{~A})$ & 1427 & 253 & 3933 & 67 \\
\hline $\mathrm{H}(19 \mathrm{~B})$ & 1463 & 388 & 3141 & 67 \\
\hline $\mathrm{H}(19 \mathrm{C})$ & 818 & 332 & 3207 & 67 \\
\hline $\mathrm{H}(15)$ & 3709 & 2465 & 755 & 40 \\
\hline $\mathrm{H}(21 \mathrm{~A})$ & 2012 & 2981 & 1077 & 56 \\
\hline $\mathrm{H}(21 \mathrm{~B})$ & 1991 & 2558 & 1711 & 56 \\
\hline $\mathrm{H}(21 \mathrm{C})$ & 1609 & 2421 & 848 & 56 \\
\hline $\mathrm{H}(42 \mathrm{~A})$ & 3520 & -593 & 4985 & 58 \\
\hline $\mathrm{H}(42 \mathrm{~B})$ & 3744 & -191 & 4476 & 58 \\
\hline $\mathrm{H}(42 \mathrm{C})$ & 3808 & -862 & 4437 & 58 \\
\hline $\mathrm{H}(25 \mathrm{~A})$ & 5210 & 991 & 3365 & 55 \\
\hline $\mathrm{H}(25 \mathrm{~B})$ & 5317 & 324 & 3374 & 55 \\
\hline $\mathrm{H}(25 \mathrm{C})$ & 5381 & 645 & 4128 & 55 \\
\hline $\mathrm{H}(18 \mathrm{~A})$ & 955 & 2390 & 2175 & 69 \\
\hline $\mathrm{H}(18 \mathrm{~B})$ & 1652 & 2509 & 2675 & 69 \\
\hline $\mathrm{H}(18 \mathrm{C})$ & 1134 & 2865 & 2810 & 69 \\
\hline $\mathrm{H}(39 \mathrm{~A})$ & 4279 & 600 & 4961 & 65 \\
\hline H(39B) & 4775 & 805 & 5745 & 65 \\
\hline $\mathrm{H}(39 \mathrm{C})$ & 4983 & 483 & 5156 & 65 \\
\hline $\mathrm{H}(8)$ & 1029 & 2608 & 3921 & 46 \\
\hline $\mathrm{H}(40 \mathrm{~A})$ & 4309 & 2345 & 2801 & 80 \\
\hline $\mathrm{H}(40 \mathrm{~B})$ & 4010 & 1730 & 2688 & 80 \\
\hline $\mathrm{H}(40 \mathrm{C})$ & 4702 & 1802 & 2786 & 80 \\
\hline $\mathrm{H}(29)$ & 5166 & 1727 & 5847 & 59 \\
\hline $\mathrm{H}(30)$ & 5259 & 2580 & 5321 & 70 \\
\hline $\mathrm{H}(31)$ & 4906 & 2683 & 4044 & 64 \\
\hline
\end{tabular}


Figure S7. Fully labeled ORTEP diagram of $\left[\mathrm{Me}_{3} \mathrm{NN}\right] \mathrm{Cu}=\mathrm{CPh}_{2}$ (8) (all $\mathrm{H}$ atoms omitted). Selected bond distances $(\AA)$, angles (deg), and twist angles between planes (deg): $\mathrm{Cu}-\mathrm{C} 24$ 1.834(3), Cu-N1 1.906(3), Cu-N2 1.922(3), N1-Cu-N2 96.10(12), C25C24-C31 117.3(3), N1-Cu-N2 / C25-C24-C31 89.0.

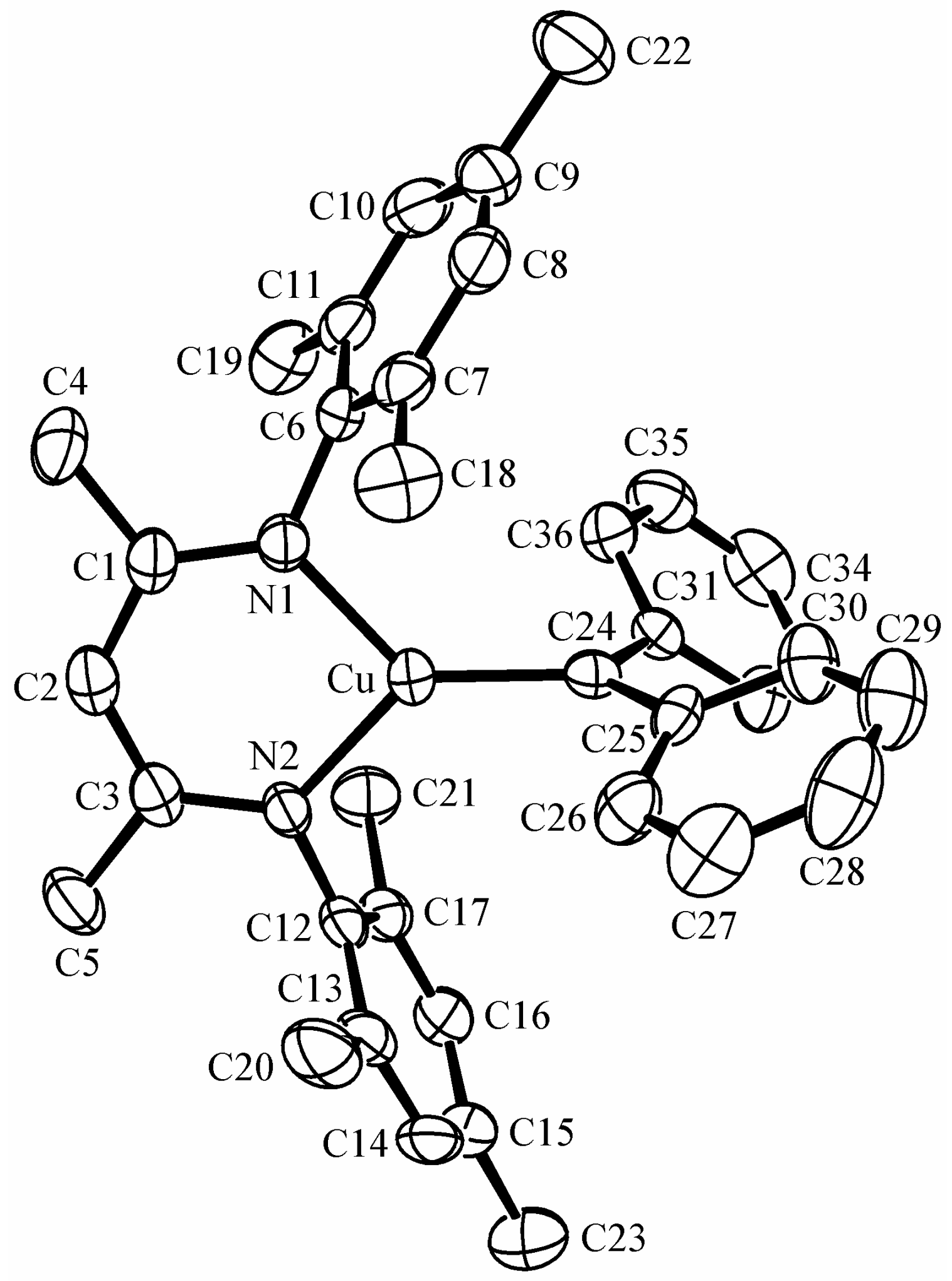


Table S6. Crystal data and structure refinedment for $\left[\mathrm{Me}_{2} \mathrm{NN}\right] \mathrm{Cu}=\mathrm{CPh}_{2}(\mathbf{8})$.

Identification code

Empirical formula

Formula weight

Temperature

Wavelength

Crystal system

Space group

Unit cell dimensions

Volume

Z

Density (calculated)

Absorption coefficient

$\mathrm{F}(000)$

Crystal size

Theta range for data collection

Index ranges

Reflections collected

Independent reflections

Completeness to theta $=26.00^{\circ}$

Absorption correction

Refinement method

Data / restraints / parameters

Goodness-of-fit on $\mathrm{F}^{2}$

Final R indices $[\mathrm{I}>2 \operatorname{sigma}(\mathrm{I})]$

$\mathrm{R}$ indices (all data)

Largest diff. peak and hole
$\left[\mathrm{Me}_{3} \mathrm{NN}\right] \mathrm{Cu}=\mathrm{CPh}_{2}(\mathbf{8})$

$\mathrm{C}_{36} \mathrm{H}_{39} \mathrm{CuN}_{2}$

563.23

173(2) K

$0.71073 \AA$

Monoclinic

$\mathrm{C} 2 / \mathrm{c}$

$$
\begin{array}{ll}
\mathrm{a}=28.733(3) \AA & \alpha=90^{\circ} . \\
\mathrm{b}=11.8213(11) \AA & \beta=112.349(2)^{\circ} . \\
\mathrm{c}=19.6365(18) \AA & \gamma=90^{\circ} .
\end{array}
$$

$6168.8(10) \AA^{3}$

8

$1.213 \mathrm{Mg} / \mathrm{m}^{3}$

$0.734 \mathrm{~mm}^{-1}$

2384

$0.16 \times 0.20 \times 0.32 \mathrm{~mm}^{3} \quad$ (violet block)

1.53 to $26.00^{\circ}$.

$-35<=\mathrm{h}<=35,-14<=\mathrm{k}<=14,-24<=1<=24$

24606

$6062[\mathrm{R}($ int $)=0.0665]$

$99.9 \%$

SADABS

Full-matrix least-squares on $\mathrm{F}^{2}$

$6062 / 0 / 360$

1.059

$\mathrm{R} 1=0.0556, \mathrm{wR} 2=0.1429$

$\mathrm{R} 1=0.0898, \mathrm{wR} 2=0.1552$

0.663 and $-0.658 \mathrm{e} \cdot \AA^{-3}$ 
Table S7. Atomic coordinates ( x 104) and equivalent isotropic displacement parameters $\left(\AA^{2} \mathrm{x}\right.$ $10^{3}$ ) for $\left[\mathrm{Me}_{2} \mathrm{NN}\right] \mathrm{Cu}=\mathrm{CPh}_{2}(\mathbf{8}) . \mathrm{U}(\mathrm{eq})$ is defined as one third of the trace of the orthogonalized $\mathrm{U}^{\mathrm{ij}}$ tensor.

\begin{tabular}{|c|c|c|c|c|}
\hline & $\mathrm{x}$ & $\mathrm{y}$ & $\mathrm{z}$ & $\mathrm{U}(\mathrm{eq})$ \\
\hline $\mathrm{Cu}(1)$ & $1337(1)$ & $2975(1)$ & $2062(1)$ & $30(1)$ \\
\hline $\mathrm{N}(2)$ & 911(1) & $1807(2)$ & $1465(2)$ & $32(1)$ \\
\hline $\mathrm{N}(1)$ & $1240(1)$ & $2596(2)$ & $2945(2)$ & $31(1)$ \\
\hline$C(12)$ & $827(1)$ & $1875(3)$ & $701(2)$ & $35(1)$ \\
\hline$C(24)$ & $1700(1)$ & 4104(3) & $1854(2)$ & $32(1)$ \\
\hline$C(6)$ & $1457(1)$ & $3400(3)$ & $3528(2)$ & $31(1)$ \\
\hline$C(3)$ & $660(1)$ & $1058(3)$ & $1701(2)$ & $35(1)$ \\
\hline$C(25)$ & $2217(1)$ & $3957(3)$ & $1917(2)$ & $33(1)$ \\
\hline $\mathrm{C}(32)$ & $1526(1)$ & $5782(3)$ & $1021(2)$ & $42(1)$ \\
\hline$C(17)$ & $486(1)$ & 2672(3) & $264(2)$ & $36(1)$ \\
\hline $\mathrm{C}(31)$ & $1466(1)$ & $5205(3)$ & $1597(2)$ & $31(1)$ \\
\hline $\mathrm{C}(11)$ & 1977(1) & $3396(3)$ & $3923(2)$ & $38(1)$ \\
\hline$C(16)$ & $425(2)$ & 2782(3) & $-465(2)$ & $44(1)$ \\
\hline$C(2)$ & $702(1)$ & $992(3)$ & $2435(2)$ & $39(1)$ \\
\hline$C(7)$ & $1157(1)$ & $4248(3)$ & $3644(2)$ & $37(1)$ \\
\hline$C(1)$ & $970(1)$ & $1724(3)$ & $3016(2)$ & $35(1)$ \\
\hline$C(36)$ & 1144(1) & $5685(3)$ & $1906(2)$ & $42(1)$ \\
\hline$C(26)$ & $2395(2)$ & $2874(3)$ & $1858(2)$ & $46(1)$ \\
\hline $\mathrm{C}(9)$ & 1902(2) & $5154(4)$ & $4507(2)$ & $54(1)$ \\
\hline$C(13)$ & $1107(1)$ & $1219(3)$ & $402(2)$ & $40(1)$ \\
\hline$C(5)$ & $304(2)$ & $251(3)$ & $1158(2)$ & $52(1)$ \\
\hline$C(10)$ & $2188(2)$ & $4295(4)$ & $4409(2)$ & $48(1)$ \\
\hline $\mathrm{C}(20)$ & $1482(2)$ & $357(3)$ & $859(3)$ & $58(1)$ \\
\hline$C(14)$ & $1030(2)$ & $1380(4)$ & $-340(2)$ & $51(1)$ \\
\hline$C(15)$ & $693(2)$ & $2143(3)$ & $-784(2)$ & $50(1)$ \\
\hline $\mathrm{C}(21)$ & 192(1) & $3413(3)$ & $585(2)$ & $46(1)$ \\
\hline$C(19)$ & $2302(2)$ & $2486(4)$ & $3803(2)$ & $55(1)$ \\
\hline$C(4)$ & $960(2)$ & $1478(4)$ & $3767(2)$ & $52(1)$ \\
\hline$C(30)$ & $2555(1)$ & $4860(3)$ & $2064(2)$ & $46(1)$ \\
\hline $\mathrm{C}(34)$ & $951(2)$ & $7230(4)$ & $1062(3)$ & $56(1)$ \\
\hline
\end{tabular}




$\begin{array}{lrrrr}\mathrm{C}(18) & 596(1) & 4266(4) & 3217(2) & 51(1) \\ \mathrm{C}(35) & 897(2) & 6685(4) & 1646(2) & 52(1) \\ \mathrm{C}(27) & 2892(2) & 2714(4) & 1938(3) & 63(1) \\ \mathrm{C}(8) & 1389(2) & 5107(3) & 4146(2) & 48(1) \\ \mathrm{C}(23) & 616(2) & 2255(4) & -1579(2) & 76(2) \\ \mathrm{C}(33) & 1263(2) & 6764(4) & 745(2) & 55(1) \\ \mathrm{C}(28) & 3215(2) & 3627(5) & 2096(3) & 72(2) \\ \mathrm{C}(29) & 3052(2) & 4694(4) & 2160(3) & 64(1) \\ \mathrm{C}(22) & 2138(2) & 6144(4) & 5010(3) & 87(2) \\ \end{array}$

Table S8. Bond lengths $[\AA]$ and angles $\left[^{\circ}\right]$ for $\left[\mathrm{Me}_{2} \mathrm{NN}\right] \mathrm{Cu}=\mathrm{CPh}_{2}(8)$.

\begin{tabular}{ll}
\hline $\mathrm{Cu}(1)-\mathrm{C}(24)$ & $1.834(3)$ \\
$\mathrm{Cu}(1)-\mathrm{N}(1)$ & $1.910(3)$ \\
$\mathrm{Cu}(1)-\mathrm{N}(2)$ & $1.919(3)$ \\
$\mathrm{N}(2)-\mathrm{C}(3)$ & $1.332(4)$ \\
$\mathrm{N}(2)-\mathrm{C}(12)$ & $1.429(4)$ \\
$\mathrm{N}(1)-\mathrm{C}(1)$ & $1.330(4)$ \\
$\mathrm{N}(1)-\mathrm{C}(6)$ & $1.435(4)$ \\
$\mathrm{C}(12)-\mathrm{C}(17)$ & $1.394(5)$ \\
$\mathrm{C}(12)-\mathrm{C}(13)$ & $1.396(5)$ \\
$\mathrm{C}(24)-\mathrm{C}(25)$ & $1.452(5)$ \\
$\mathrm{C}(24)-\mathrm{C}(31)$ & $1.464(5)$ \\
$\mathrm{C}(6)-\mathrm{C}(7)$ & $1.396(5)$ \\
$\mathrm{C}(6)-\mathrm{C}(11)$ & $1.396(5)$ \\
$\mathrm{C}(3)-\mathrm{C}(2)$ & $1.402(5)$ \\
$\mathrm{C}(3)-\mathrm{C}(5)$ & $1.504(5)$ \\
$\mathrm{C}(25)-\mathrm{C}(30)$ & $1.399(5)$ \\
$\mathrm{C}(25)-\mathrm{C}(26)$ & $1.399(5)$ \\
$\mathrm{C}(32)-\mathrm{C}(33)$ & $1.379(5)$ \\
$\mathrm{C}(32)-\mathrm{C}(31)$ & $1.386(5)$ \\
$\mathrm{C}(17)-\mathrm{C}(16)$ & $1.380(5)$ \\
$\mathrm{C}(17)-\mathrm{C}(21)$ & $1.511(5)$ \\
$\mathrm{C}(31)-\mathrm{C}(36)$ & $1.402(5)$ \\
$\mathrm{C}(11)-\mathrm{C}(10)$ & $1.404(5)$ \\
&
\end{tabular}




\begin{tabular}{|c|c|}
\hline C(11)-C(19) & $1.502(5)$ \\
\hline$C(16)-C(15)$ & $1.387(5)$ \\
\hline$C(2)-C(1)$ & $1.406(5)$ \\
\hline$C(7)-C(8)$ & $1.395(5)$ \\
\hline$C(7)-C(18)$ & $1.510(5)$ \\
\hline$C(1)-C(4)$ & $1.515(5)$ \\
\hline$C(36)-C(35)$ & $1.373(5)$ \\
\hline$C(26)-C(27)$ & $1.388(6)$ \\
\hline$C(9)-C(10)$ & $1.365(6)$ \\
\hline$C(9)-C(8)$ & $1.371(6)$ \\
\hline$C(9)-C(22)$ & $1.515(6)$ \\
\hline$C(13)-C(14)$ & $1.400(5)$ \\
\hline$C(13)-C(20)$ & $1.508(6)$ \\
\hline$C(14)-C(15)$ & $1.367(6)$ \\
\hline$C(15)-C(23)$ & $1.497(6)$ \\
\hline$C(30)-C(29)$ & $1.381(5)$ \\
\hline$C(34)-C(35)$ & $1.376(6)$ \\
\hline$C(34)-C(33)$ & $1.383(6)$ \\
\hline $\mathrm{C}(27)-\mathrm{C}(28)$ & $1.381(7)$ \\
\hline$C(28)-C(29)$ & $1.367(7)$ \\
\hline $\mathrm{C}(24)-\mathrm{Cu}(1)-\mathrm{N}(1)$ & $131.55(13)$ \\
\hline $\mathrm{C}(24)-\mathrm{Cu}(1)-\mathrm{N}(2)$ & $132.30(13)$ \\
\hline $\mathrm{N}(1)-\mathrm{Cu}(1)-\mathrm{N}(2)$ & $96.12(12)$ \\
\hline $\mathrm{C}(3)-\mathrm{N}(2)-\mathrm{C}(12)$ & $120.3(3)$ \\
\hline $\mathrm{C}(3)-\mathrm{N}(2)-\mathrm{Cu}(1)$ & $124.7(2)$ \\
\hline $\mathrm{C}(12)-\mathrm{N}(2)-\mathrm{Cu}(1)$ & $114.5(2)$ \\
\hline$C(1)-N(1)-C(6)$ & $121.5(3)$ \\
\hline $\mathrm{C}(1)-\mathrm{N}(1)-\mathrm{Cu}(1)$ & $124.7(2)$ \\
\hline $\mathrm{C}(6)-\mathrm{N}(1)-\mathrm{Cu}(1)$ & $113.6(2)$ \\
\hline$C(17)-C(12)-C(13)$ & $119.9(3)$ \\
\hline $\mathrm{C}(17)-\mathrm{C}(12)-\mathrm{N}(2)$ & $118.8(3)$ \\
\hline $\mathrm{C}(13)-\mathrm{C}(12)-\mathrm{N}(2)$ & $121.1(3)$ \\
\hline$C(25)-C(24)-C(31)$ & 117.2(3) \\
\hline $\mathrm{C}(25)-\mathrm{C}(24)-\mathrm{Cu}(1)$ & $122.9(3)$ \\
\hline $\mathrm{C}(31)-\mathrm{C}(24)-\mathrm{Cu}(1)$ & $119.8(2)$ \\
\hline
\end{tabular}




\begin{tabular}{|c|c|}
\hline$C(7)-C(6)-C(11)$ & $120.4(3)$ \\
\hline $\mathrm{C}(7)-\mathrm{C}(6)-\mathrm{N}(1)$ & $119.9(3)$ \\
\hline $\mathrm{C}(11)-\mathrm{C}(6)-\mathrm{N}(1)$ & $119.3(3)$ \\
\hline $\mathrm{N}(2)-\mathrm{C}(3)-\mathrm{C}(2)$ & $123.3(3)$ \\
\hline $\mathrm{N}(2)-\mathrm{C}(3)-\mathrm{C}(5)$ & $119.2(3)$ \\
\hline $\mathrm{C}(2)-\mathrm{C}(3)-\mathrm{C}(5)$ & $117.6(3)$ \\
\hline$C(30)-C(25)-C(26)$ & $117.9(3)$ \\
\hline$C(30)-C(25)-C(24)$ & $122.3(3)$ \\
\hline$C(26)-C(25)-C(24)$ & $119.8(3)$ \\
\hline$C(33)-C(32)-C(31)$ & $121.0(4)$ \\
\hline$C(16)-C(17)-C(12)$ & $119.0(3)$ \\
\hline$C(16)-C(17)-C(21)$ & $120.4(3)$ \\
\hline$C(12)-C(17)-C(21)$ & $120.6(3)$ \\
\hline$C(32)-C(31)-C(36)$ & $117.4(3)$ \\
\hline$C(32)-C(31)-C(24)$ & $122.3(3)$ \\
\hline$C(36)-C(31)-C(24)$ & $120.2(3)$ \\
\hline$C(6)-C(11)-C(10)$ & $118.0(4)$ \\
\hline$C(6)-C(11)-C(19)$ & $120.9(3)$ \\
\hline$C(10)-C(11)-C(19)$ & $121.0(4)$ \\
\hline$C(17)-C(16)-C(15)$ & $122.9(4)$ \\
\hline$C(3)-C(2)-C(1)$ & $127.3(3)$ \\
\hline$C(8)-C(7)-C(6)$ & $118.7(3)$ \\
\hline$C(8)-C(7)-C(18)$ & $120.3(3)$ \\
\hline$C(6)-C(7)-C(18)$ & $121.0(3)$ \\
\hline $\mathrm{N}(1)-\mathrm{C}(1)-\mathrm{C}(2)$ & $123.6(3)$ \\
\hline $\mathrm{N}(1)-\mathrm{C}(1)-\mathrm{C}(4)$ & $118.7(3)$ \\
\hline$C(2)-C(1)-C(4)$ & $117.6(3)$ \\
\hline$C(35)-C(36)-C(31)$ & $121.4(4)$ \\
\hline$C(27)-C(26)-C(25)$ & $120.6(4)$ \\
\hline$C(10)-C(9)-C(8)$ & $118.9(4)$ \\
\hline$C(10)-C(9)-C(22)$ & $121.4(5)$ \\
\hline $\mathrm{C}(8)-\mathrm{C}(9)-\mathrm{C}(22)$ & $119.7(5)$ \\
\hline$C(12)-C(13)-C(14)$ & $118.3(4)$ \\
\hline$C(12)-C(13)-C(20)$ & 121.3(4) \\
\hline$C(14)-C(13)-C(20)$ & $120.4(3)$ \\
\hline$C(9)-C(10)-C(11)$ & $122.1(4)$ \\
\hline
\end{tabular}




$\begin{array}{ll}\mathrm{C}(15)-\mathrm{C}(14)-\mathrm{C}(13) & 123.1(4) \\ \mathrm{C}(14)-\mathrm{C}(15)-\mathrm{C}(16) & 116.8(4) \\ \mathrm{C}(14)-\mathrm{C}(15)-\mathrm{C}(23) & 120.7(4) \\ \mathrm{C}(16)-\mathrm{C}(15)-\mathrm{C}(23) & 122.4(4) \\ \mathrm{C}(29)-\mathrm{C}(30)-\mathrm{C}(25) & 121.4(4) \\ \mathrm{C}(35)-\mathrm{C}(34)-\mathrm{C}(33) & 119.1(4) \\ \mathrm{C}(36)-\mathrm{C}(35)-\mathrm{C}(34) & 120.4(4) \\ \mathrm{C}(28)-\mathrm{C}(27)-\mathrm{C}(26) & 119.5(4) \\ \mathrm{C}(9)-\mathrm{C}(8)-\mathrm{C}(7) & 121.7(4) \\ \mathrm{C}(32)-\mathrm{C}(33)-\mathrm{C}(34) & 120.7(4) \\ \mathrm{C}(29)-\mathrm{C}(28)-\mathrm{C}(27) & 121.2(4) \\ \mathrm{C}(28)-\mathrm{C}(29)-\mathrm{C}(30) & 119.4(4)\end{array}$

Table S9. Anisotropic displacement parameters $\left(\AA^{2} \times 10^{3}\right)$ for $\left[\mathrm{Me}_{2} \mathrm{NN}\right] \mathrm{Cu}=\mathrm{CPh}_{2}(8)$. The anisotropic displacement factor exponent takes the form: $-2 \pi^{2}\left[\mathrm{~h}^{2} \mathrm{a}^{* 2} \mathrm{U}^{11}+\ldots+2 \mathrm{~h} \mathrm{k} \mathrm{a*} \mathrm{b}^{*}\right.$ $\left.\mathrm{U}^{12}\right]$

\begin{tabular}{lllllll}
\hline & $\mathrm{U}^{11}$ & $\mathrm{U}^{22}$ & $\mathrm{U}^{33}$ & $\mathrm{U}^{23}$ & $\mathrm{U}^{13}$ & $\mathrm{U}^{12}$ \\
\hline $\mathrm{Cu}(1)$ & $31(1)$ & $28(1)$ & $33(1)$ & $0(1)$ & $14(1)$ & $-6(1)$ \\
$\mathrm{N}(2)$ & $32(2)$ & $25(2)$ & $42(2)$ & $-6(1)$ & $18(1)$ & $-6(1)$ \\
$\mathrm{N}(1)$ & $32(2)$ & $29(2)$ & $34(2)$ & $1(1)$ & $14(1)$ & $-2(1)$ \\
$\mathrm{C}(12)$ & $34(2)$ & $30(2)$ & $44(2)$ & $-13(2)$ & $20(2)$ & $-13(2)$ \\
$\mathrm{C}(24)$ & $38(2)$ & $35(2)$ & $23(2)$ & $-5(1)$ & $10(2)$ & $-6(2)$ \\
$\mathrm{C}(6)$ & $38(2)$ & $28(2)$ & $28(2)$ & $3(1)$ & $15(2)$ & $-5(2)$ \\
$\mathrm{C}(3)$ & $27(2)$ & $27(2)$ & $52(2)$ & $-2(2)$ & $18(2)$ & $1(2)$ \\
$\mathrm{C}(25)$ & $33(2)$ & $40(2)$ & $27(2)$ & $8(2)$ & $14(2)$ & $-1(2)$ \\
$\mathrm{C}(32)$ & $41(2)$ & $42(2)$ & $42(2)$ & $7(2)$ & $16(2)$ & $-2(2)$ \\
$\mathrm{C}(17)$ & $30(2)$ & $40(2)$ & $37(2)$ & $-9(2)$ & $13(2)$ & $-8(2)$ \\
$\mathrm{C}(31)$ & $31(2)$ & $32(2)$ & $30(2)$ & $-5(2)$ & $9(2)$ & $-7(2)$ \\
$\mathrm{C}(11)$ & $40(2)$ & $47(2)$ & $27(2)$ & $3(2)$ & $12(2)$ & $-3(2)$ \\
$\mathrm{C}(16)$ & $46(2)$ & $45(2)$ & $40(2)$ & $-6(2)$ & $15(2)$ & $-10(2)$ \\
$\mathrm{C}(2)$ & $34(2)$ & $31(2)$ & $55(2)$ & $2(2)$ & $21(2)$ & $-2(2)$ \\
$\mathrm{C}(7)$ & $48(2)$ & $35(2)$ & $36(2)$ & $7(2)$ & $24(2)$ & $4(2)$ \\
$\mathrm{C}(1)$ & $36(2)$ & $29(2)$ & $46(2)$ & $5(2)$ & $21(2)$ & $0(2)$ \\
& & & & & & \\
& & & & & \\
& & & & & \\
\end{tabular}




\begin{tabular}{lllllll}
$\mathrm{C}(36)$ & $40(2)$ & $46(2)$ & $41(2)$ & $-4(2)$ & $17(2)$ & $-9(2)$ \\
$\mathrm{C}(26)$ & $53(3)$ & $43(2)$ & $44(2)$ & $13(2)$ & $23(2)$ & $7(2)$ \\
$\mathrm{C}(9)$ & $85(3)$ & $42(2)$ & $35(2)$ & $-3(2)$ & $22(2)$ & $-19(2)$ \\
$\mathrm{C}(13)$ & $35(2)$ & $35(2)$ & $52(2)$ & $-16(2)$ & $18(2)$ & $-8(2)$ \\
$\mathrm{C}(5)$ & $51(2)$ & $38(2)$ & $69(3)$ & $-16(2)$ & $26(2)$ & $-16(2)$ \\
$\mathrm{C}(10)$ & $47(2)$ & $61(3)$ & $35(2)$ & $1(2)$ & $15(2)$ & $-17(2)$ \\
$\mathrm{C}(20)$ & $55(3)$ & $44(2)$ & $83(3)$ & $-17(2)$ & $34(2)$ & $4(2)$ \\
$\mathrm{C}(14)$ & $52(3)$ & $54(3)$ & $61(3)$ & $-30(2)$ & $37(2)$ & $-16(2)$ \\
$\mathrm{C}(15)$ & $61(3)$ & $51(3)$ & $43(2)$ & $-16(2)$ & $27(2)$ & $-20(2)$ \\
$\mathrm{C}(21)$ & $44(2)$ & $49(2)$ & $46(2)$ & $-3(2)$ & $17(2)$ & $7(2)$ \\
$\mathrm{C}(19)$ & $37(2)$ & $69(3)$ & $53(3)$ & $1(2)$ & $11(2)$ & $11(2)$ \\
$\mathrm{C}(4)$ & $66(3)$ & $44(2)$ & $53(3)$ & $14(2)$ & $32(2)$ & $-5(2)$ \\
$\mathrm{C}(30)$ & $34(2)$ & $47(2)$ & $51(2)$ & $9(2)$ & $10(2)$ & $-4(2)$ \\
$\mathrm{C}(34)$ & $46(3)$ & $42(2)$ & $69(3)$ & $3(2)$ & $9(2)$ & $6(2)$ \\
$\mathrm{C}(18)$ & $49(2)$ & $52(3)$ & $63(3)$ & $10(2)$ & $32(2)$ & $15(2)$ \\
$\mathrm{C}(35)$ & $47(2)$ & $47(2)$ & $60(3)$ & $-13(2)$ & $20(2)$ & $1(2)$ \\
$\mathrm{C}(27)$ & $61(3)$ & $61(3)$ & $74(3)$ & $24(2)$ & $32(3)$ & $26(2)$ \\
$\mathrm{C}(8)$ & $76(3)$ & $37(2)$ & $42(2)$ & $3(2)$ & $34(2)$ & $9(2)$ \\
$\mathrm{C}(23)$ & $99(4)$ & $87(4)$ & $52(3)$ & $-22(3)$ & $39(3)$ & $-22(3)$ \\
$\mathrm{C}(33)$ & $54(3)$ & $47(3)$ & $61(3)$ & $21(2)$ & $20(2)$ & $2(2)$ \\
$\mathrm{C}(28)$ & $40(3)$ & $98(4)$ & $80(4)$ & $37(3)$ & $25(2)$ & $11(3)$ \\
$\mathrm{C}(29)$ & $32(2)$ & $78(3)$ & $75(3)$ & $16(3)$ & $14(2)$ & $-7(2)$ \\
$\mathrm{C}(22)$ & $145(5)$ & $55(3)$ & $58(3)$ & $-13(2)$ & $35(3)$ & $-36(3)$ \\
& & & & & & \\
\hline
\end{tabular}


Table S10. Hydrogen coordinates $\left(\mathrm{x} 10^{4}\right)$ and isotropic displacement parameters $\left(\AA^{2} \times 10^{3}\right)$ for $\left[\mathrm{Me}_{2} \mathrm{NN}\right] \mathrm{Cu}=\mathrm{CPh}_{2}(8)$.

\begin{tabular}{|c|c|c|c|c|}
\hline & $\mathrm{x}$ & $\mathrm{y}$ & $\mathrm{z}$ & $\mathrm{U}(\mathrm{eq})$ \\
\hline $\mathrm{H}(32)$ & 1752 & 5496 & 814 & 50 \\
\hline $\mathrm{H}(16)$ & 188 & 3319 & -761 & 53 \\
\hline $\mathrm{H}(36)$ & 1097 & 5310 & 2302 & 50 \\
\hline $\mathrm{H}(26)$ & 2174 & 2244 & 1763 & 55 \\
\hline $\mathrm{H}(5 \mathrm{~A})$ & 25 & 676 & 801 & 77 \\
\hline $\mathrm{H}(5 \mathrm{~B})$ & 172 & -286 & 1420 & 77 \\
\hline $\mathrm{H}(5 \mathrm{C})$ & 484 & -162 & 900 & 77 \\
\hline $\mathrm{H}(10)$ & 2541 & 4306 & 4680 & 58 \\
\hline $\mathrm{H}(20 \mathrm{~A})$ & 1464 & -320 & 562 & 87 \\
\hline $\mathrm{H}(20 \mathrm{~B})$ & 1405 & 153 & 1289 & 87 \\
\hline $\mathrm{H}(20 \mathrm{C})$ & 1822 & 676 & 1025 & 87 \\
\hline $\mathrm{H}(14)$ & 1221 & 939 & -544 & 61 \\
\hline $\mathrm{H}(21 \mathrm{~A})$ & -72 & 3814 & 187 & 69 \\
\hline $\mathrm{H}(21 \mathrm{~B})$ & 420 & 3964 & 923 & 69 \\
\hline $\mathrm{H}(21 \mathrm{C})$ & 38 & 2943 & 854 & 69 \\
\hline $\mathrm{H}(19 \mathrm{~A})$ & 2174 & 1743 & 3871 & 82 \\
\hline H(19B) & 2648 & 2578 & 4159 & 82 \\
\hline $\mathrm{H}(19 \mathrm{C})$ & 2296 & 2543 & 3302 & 82 \\
\hline $\mathrm{H}(4 \mathrm{~A})$ & 1305 & 1427 & 4132 & 78 \\
\hline $\mathrm{H}(4 \mathrm{~B})$ & 785 & 760 & 3751 & 78 \\
\hline $\mathrm{H}(4 \mathrm{C})$ & 782 & 2089 & 3905 & 78 \\
\hline $\mathrm{H}(30)$ & 2441 & 5604 & 2098 & 55 \\
\hline $\mathrm{H}(34)$ & 777 & 7917 & 878 & 67 \\
\hline $\mathrm{H}(18 \mathrm{~A})$ & 445 & 4874 & 3404 & 77 \\
\hline $\mathrm{H}(18 \mathrm{~B})$ & 451 & 3538 & 3275 & 77 \\
\hline $\mathrm{H}(18 \mathrm{C})$ & 526 & 4397 & 2695 & 77 \\
\hline $\mathrm{H}(35)$ & 688 & 7002 & 1872 & 62 \\
\hline $\mathrm{H}(27)$ & 3008 & 1980 & 1883 & 76 \\
\hline $\mathrm{H}(8)$ & 1187 & 5673 & 4240 & 58 \\
\hline $\mathrm{H}(23 \mathrm{~A})$ & 886 & 2718 & -1622 & 114 \\
\hline
\end{tabular}




\begin{tabular}{lrrrr}
$\mathrm{H}(23 \mathrm{~B})$ & 291 & 2617 & -1848 & 114 \\
$\mathrm{H}(23 \mathrm{C})$ & 621 & 1503 & -1786 & 114 \\
$\mathrm{H}(33)$ & 1296 & 7124 & 334 & 66 \\
$\mathrm{H}(28)$ & 3557 & 3513 & 2161 & 86 \\
$\mathrm{H}(29)$ & 3279 & 5315 & 2271 & 77 \\
$\mathrm{H}(22 \mathrm{~A})$ & 2252 & 6708 & 4742 & 130 \\
$\mathrm{H}(22 \mathrm{~B})$ & 2426 & 5878 & 5438 & 130 \\
$\mathrm{H}(22 \mathrm{C})$ & 1888 & 6486 & 5176 & 130 \\
& & & & \\
\hline
\end{tabular}

\section{Selected Calculation Output for $\left[\mathrm{H}_{5} \mathrm{C}_{3} \mathrm{~N}_{2}\right] \mathrm{Cu}=\mathrm{CH}_{2}(6)$}

TITLE mini $\mathrm{Cu}=\mathrm{CH} 2$ relativistic zora

SYMMETRY C(2V)

MAXMEMORYUSAGE 330

REALMEMBLOCK 10

INTEGERMEMBLOCK 5

LOGICALMEMBLOCK 1

STRINGMEMBLOCK 15

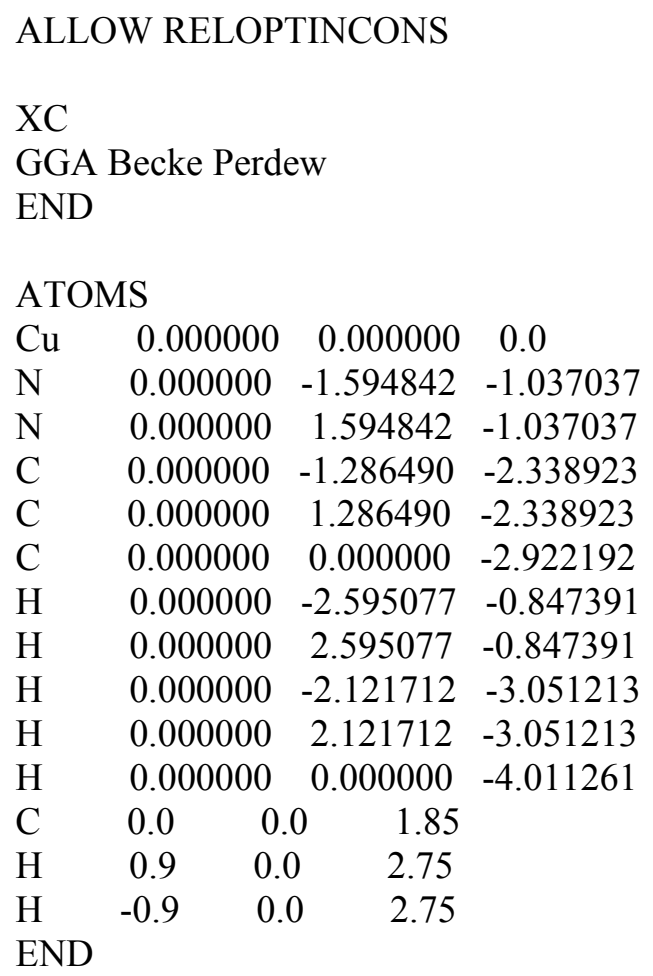

Fragments 
$\mathrm{H}$ t21.H

$\mathrm{N}$ t21.N

$\mathrm{Cu}$ t21.Cu

C t21.C

End

Relativistic scalar ZORA

CorePotentials t12.rel \&

$\mathrm{Cu} 1$

C 2

H 3

N 4

END

GEOMETRY

END

END INPUT

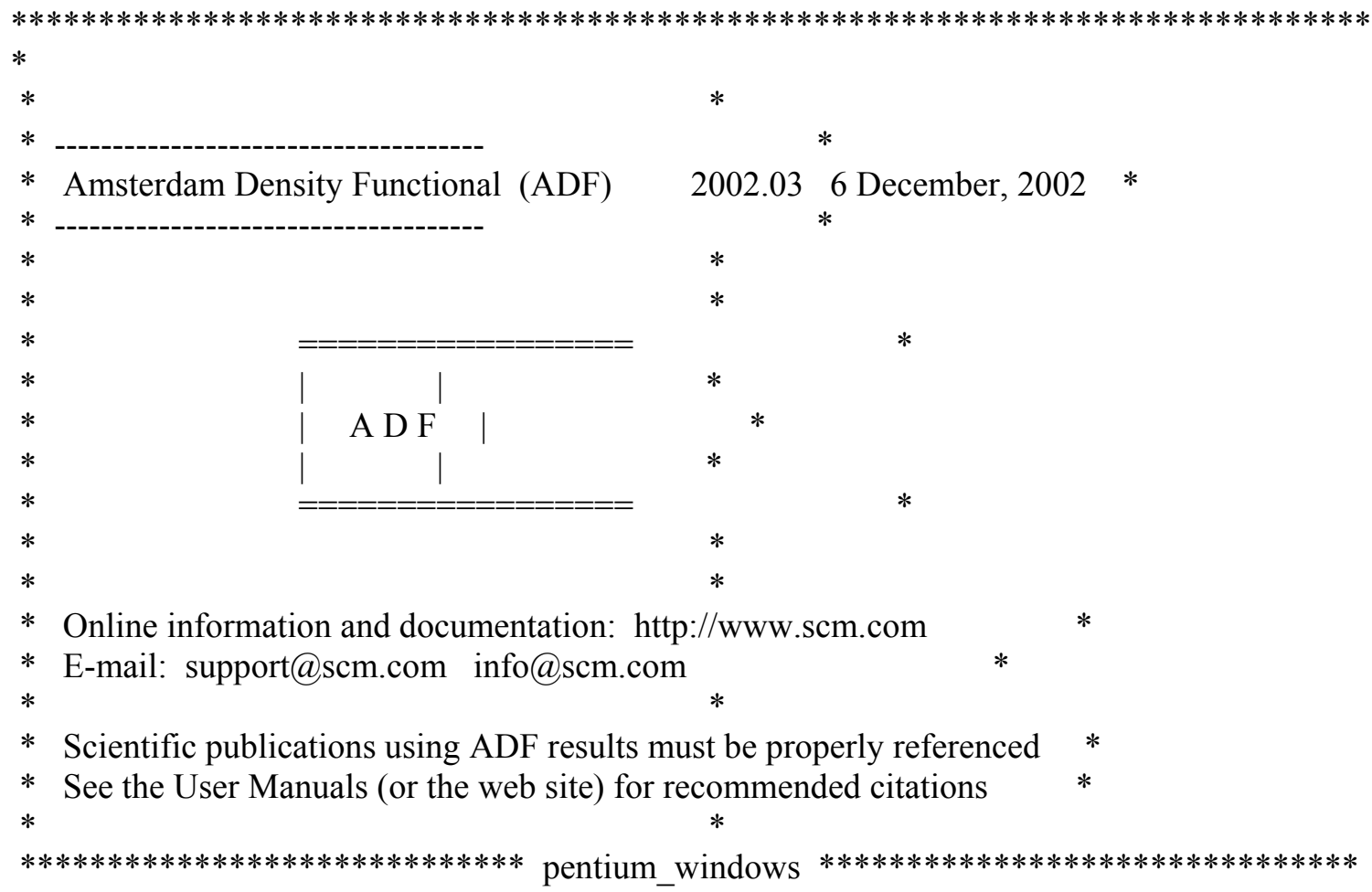

ADF 2002.03 RunTime: Jan04-2004 09:46:22 mini $\mathrm{Cu}=\mathrm{CH} 2$ relativistic zora

\section{A T T A C H E D F I L E S}


Core Potentials:

file : t12.rel

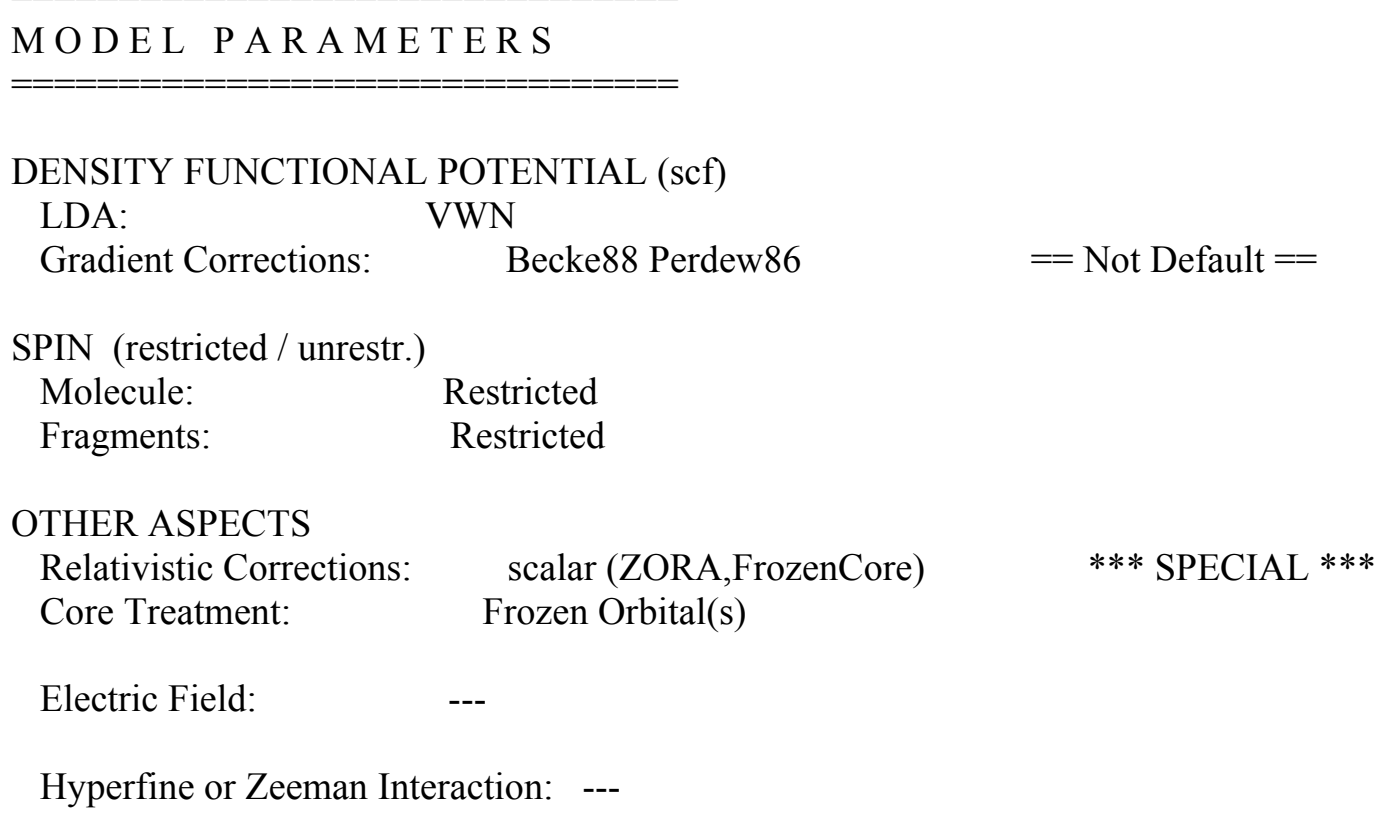

$\mathrm{Cu}:$

file : $21 . \mathrm{Cu}$

jobid: ADF 2002.03 RunTime: Dec31-2003 22:59:20

title: Copper (TZ2P+, $2 p$ frozen) $\mathrm{N}$ :

file : t21.N

jobid: ADF 2002.03 RunTime: Dec31-2003 23:02:35

title: Nitrogen (V, 1s frozen)

$\mathrm{C}$ :

file : t21.C

jobid: ADF 2002.03 RunTime: Dec31-2003 23:01:27

title: Carbon (V, 1s frozen)

$\mathrm{H}$ :

file : $\mathrm{t} 21 . \mathrm{H}$

jobid: ADF 2002.03 RunTime: Dec31-2003 23:00:18

title: Hydrogen (V)

$* * * * * * * * * * * * * * * * * * * * * * * * * * * * * * * * * * * * * * * * * * * * *$

* R U N T Y P E : GEOMETRY OPTIMIZATION *

$* * * * * * * * * * * * * * * * * * * * * * * * * * * * * * * * * * * * * * * * * * * * *$ 


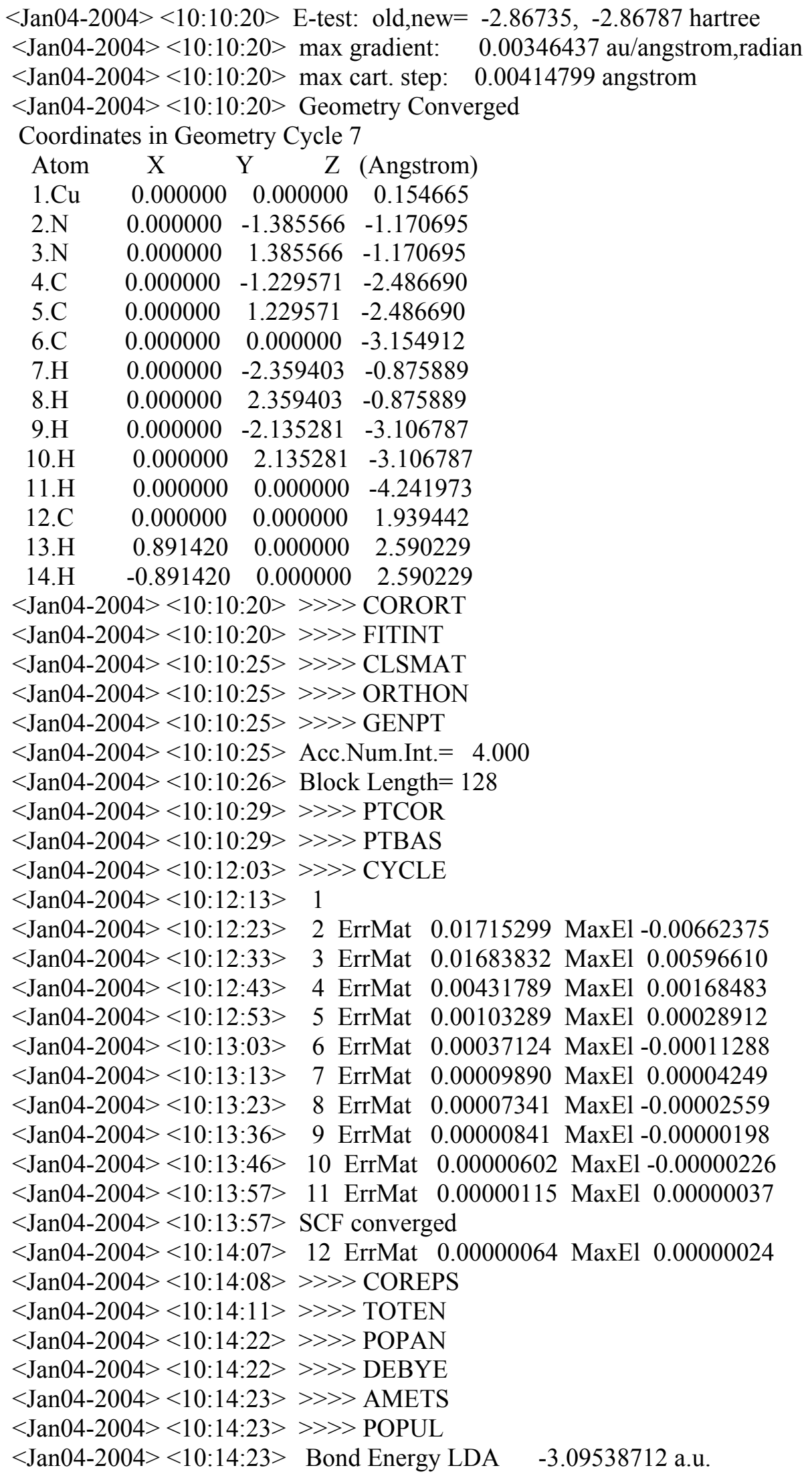




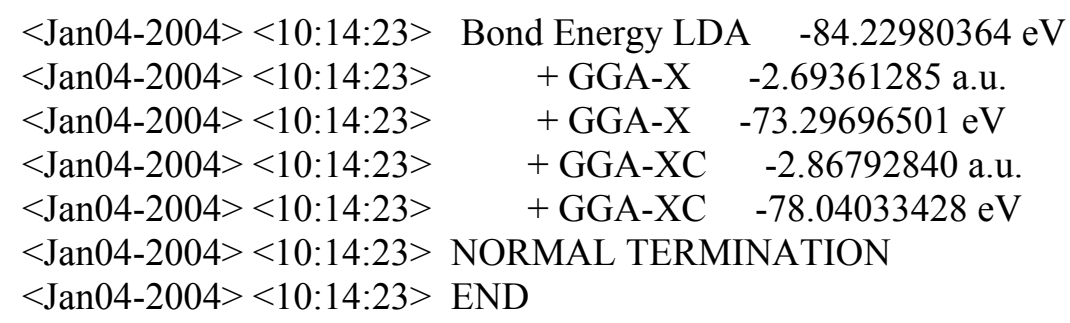

\section{Selected Calculation Output for $\left\{\left[\mathrm{H}_{5} \mathrm{C}_{3} \mathrm{~N}_{2}\right] \mathrm{Cu}\right\}_{2}\left(\mu-\mathrm{CH}_{2}\right)\left(7-\mathrm{C}_{2 \mathrm{v}}\right)$}

TITLE model $\mathrm{Cu} 2 \mathrm{CH} 2$

SYMMETRY C(2V)

MAXMEMORYUSAGE 330

REALMEMBLOCK 10

INTEGERMEMBLOCK 5

LOGICALMEMBLOCK 1

STRINGMEMBLOCK 15

$\mathrm{XC}$

GGA Becke Perdew

END

\section{ALLOW RELOPTINCONS}

$\begin{array}{lrrl}\text { ATOMS } & & & \\ \mathrm{C} & 0.000000 & 0.000000 & -0.102917 \\ \mathrm{H} & 0.862797 & 0.000000 & 0.599826 \\ \mathrm{H} & -0.862797 & 0.000000 & 0.599826 \\ \mathrm{Cu} & -1.266817 & 0.000000 & -1.504607 \\ \mathrm{~N} & -2.230572 & 1.432290 & -2.381146 \\ \mathrm{~N} & -2.230572 & -1.432290 & -2.381146 \\ \mathrm{C} & -3.159930 & 1.242602 & -3.306919 \\ \mathrm{C} & -3.159930 & -1.242602 & -3.306919 \\ \mathrm{C} & -3.621529 & 0.000000 & -3.763468 \\ \mathrm{H} & -2.028899 & -2.415315 & -2.210136 \\ \mathrm{H} & -2.028899 & 2.415315 & -2.210136 \\ \mathrm{H} & -3.612183 & -2.131479 & -3.767571 \\ \mathrm{H} & -3.612183 & 2.131479 & -3.767571 \\ \mathrm{H} & -4.385772 & 0.000000 & -4.537218 \\ \mathrm{Cu} & 1.266817 & 0.000000 & -1.504607 \\ \mathrm{~N} & 2.230572 & 1.432290 & -2.381146 \\ \mathrm{~N} & 2.230572 & -1.432290 & -2.381146 \\ \mathrm{C} & 3.159930 & 1.242602 & -3.306919 \\ \mathrm{C} & 3.159930 & -1.242602 & -3.306919 \\ \mathrm{C} & 3.621529 & 0.000000 & -3.763468 \\ \mathrm{H} & 2.028899 & -2.415315 & -2.210136 \\ \mathrm{H} & 2.028899 & 2.415315 & -2.210136\end{array}$




$\begin{array}{lrrr}\mathrm{H} & 3.612183 & -2.131479 & -3.767571 \\ \mathrm{H} & 3.612183 & 2.131479 & -3.767571 \\ \mathrm{H} & 4.385772 & 0.000000 & -4.537218 \\ \text { END } & & & \end{array}$

Fragments

$\mathrm{H}$ t21.H

$\mathrm{N}$ t21.N

$\mathrm{Cu} \mathrm{t} 21 . \mathrm{Cu}$

C t21.C

End

Relativistic scalar ZORA

CorePotentials t12.rel \&

$\mathrm{Cu} 1$

C 2

H 3

N 4

END

\section{GEOMETRY}

End

\section{END INPUT}

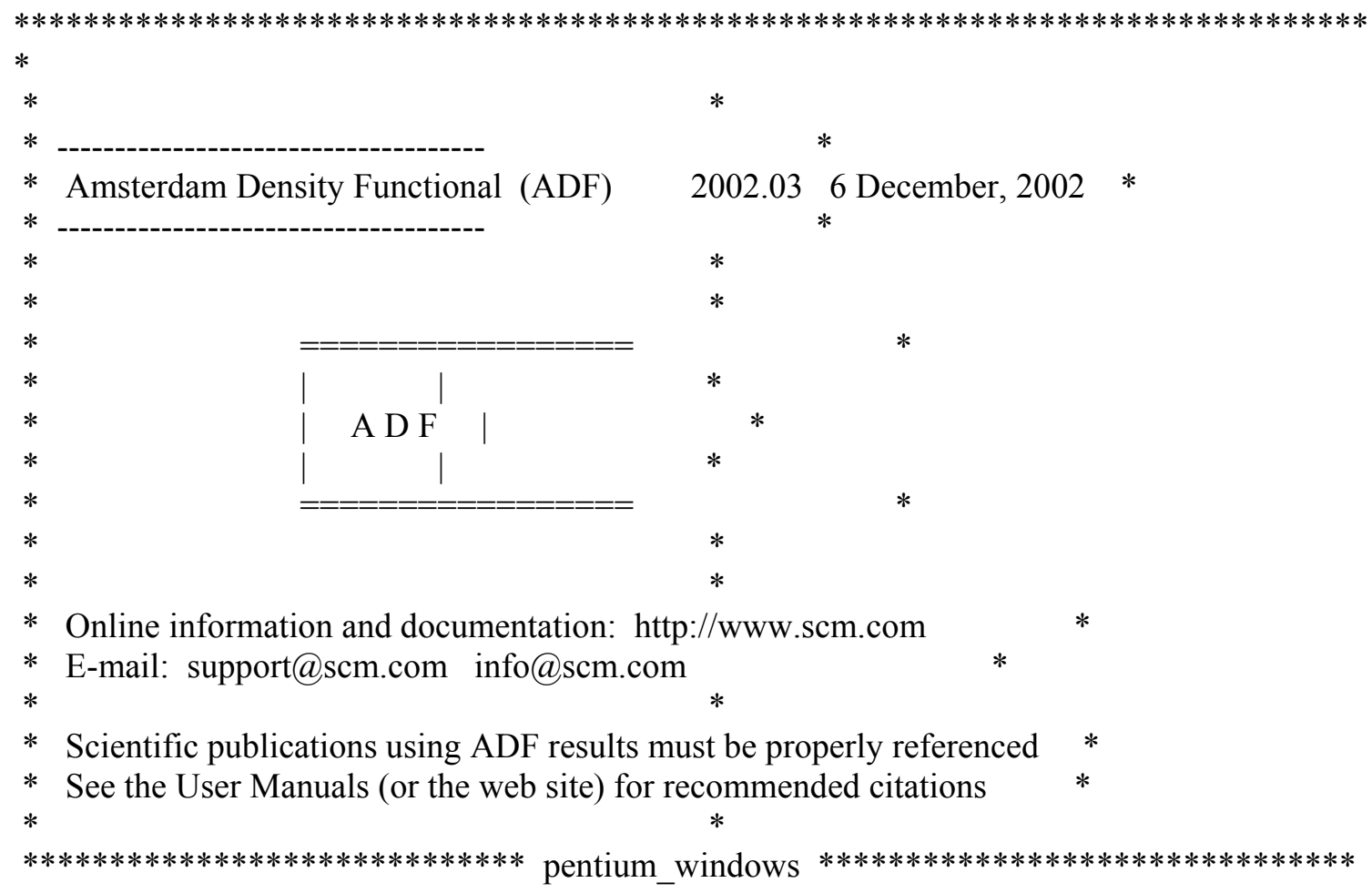

ADF 2002.03 RunTime: Dec31-2003 23:25:14 model $\mathrm{Cu} 2 \mathrm{CH} 2$ 
A T T A C HE D F I L E S

Core Potentials:

file : t12.rel

MODEL PARAMETERS

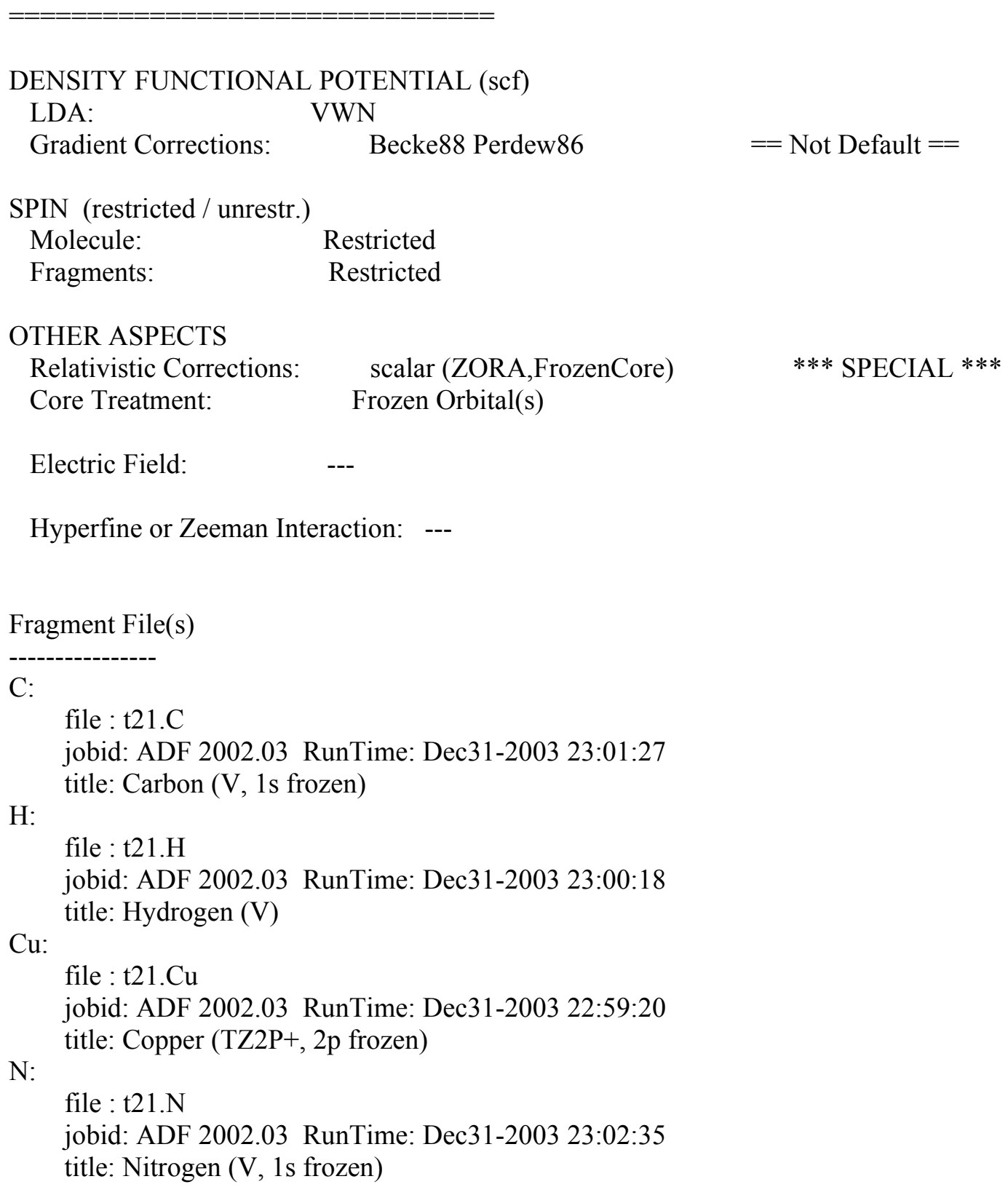




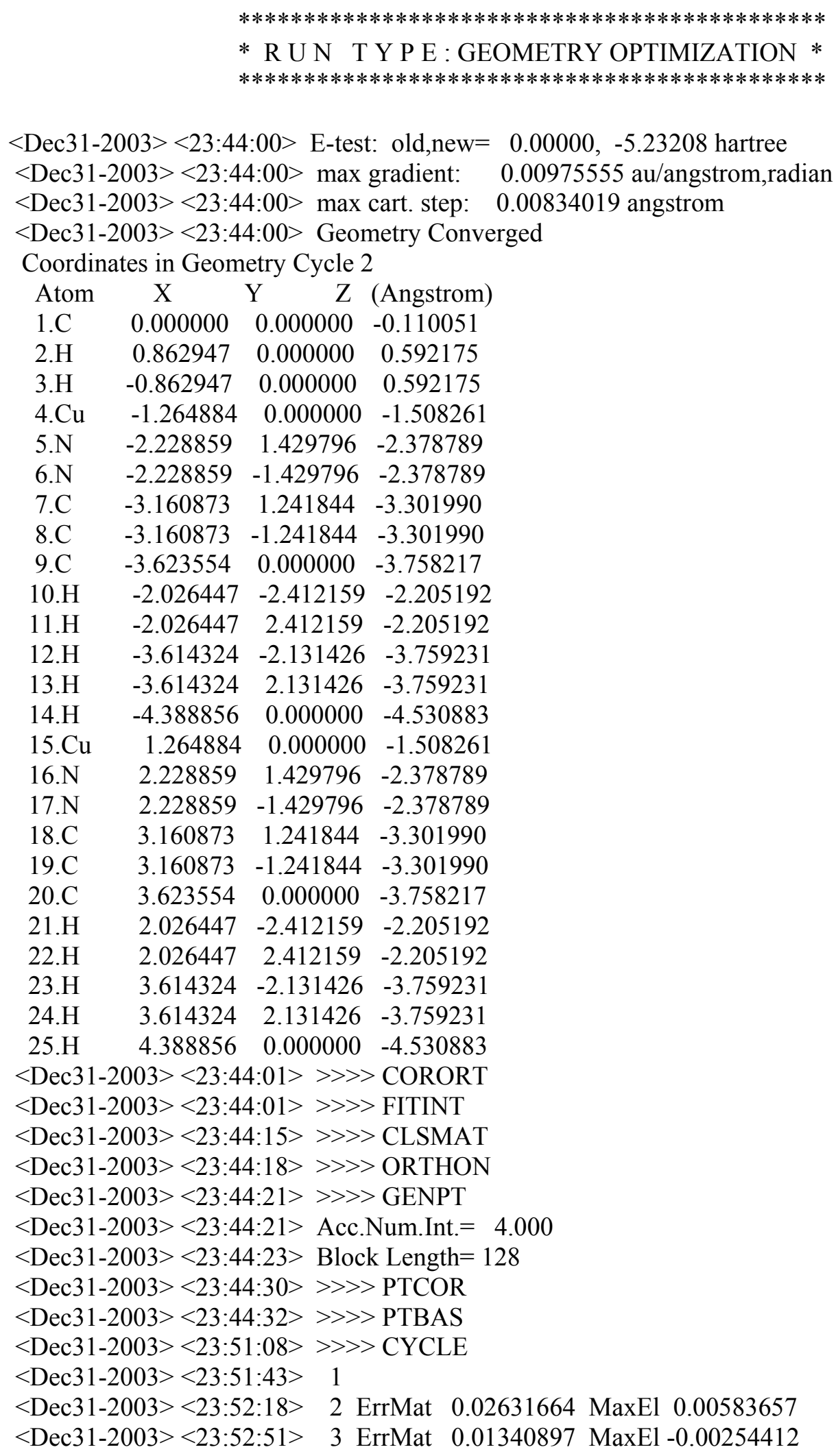




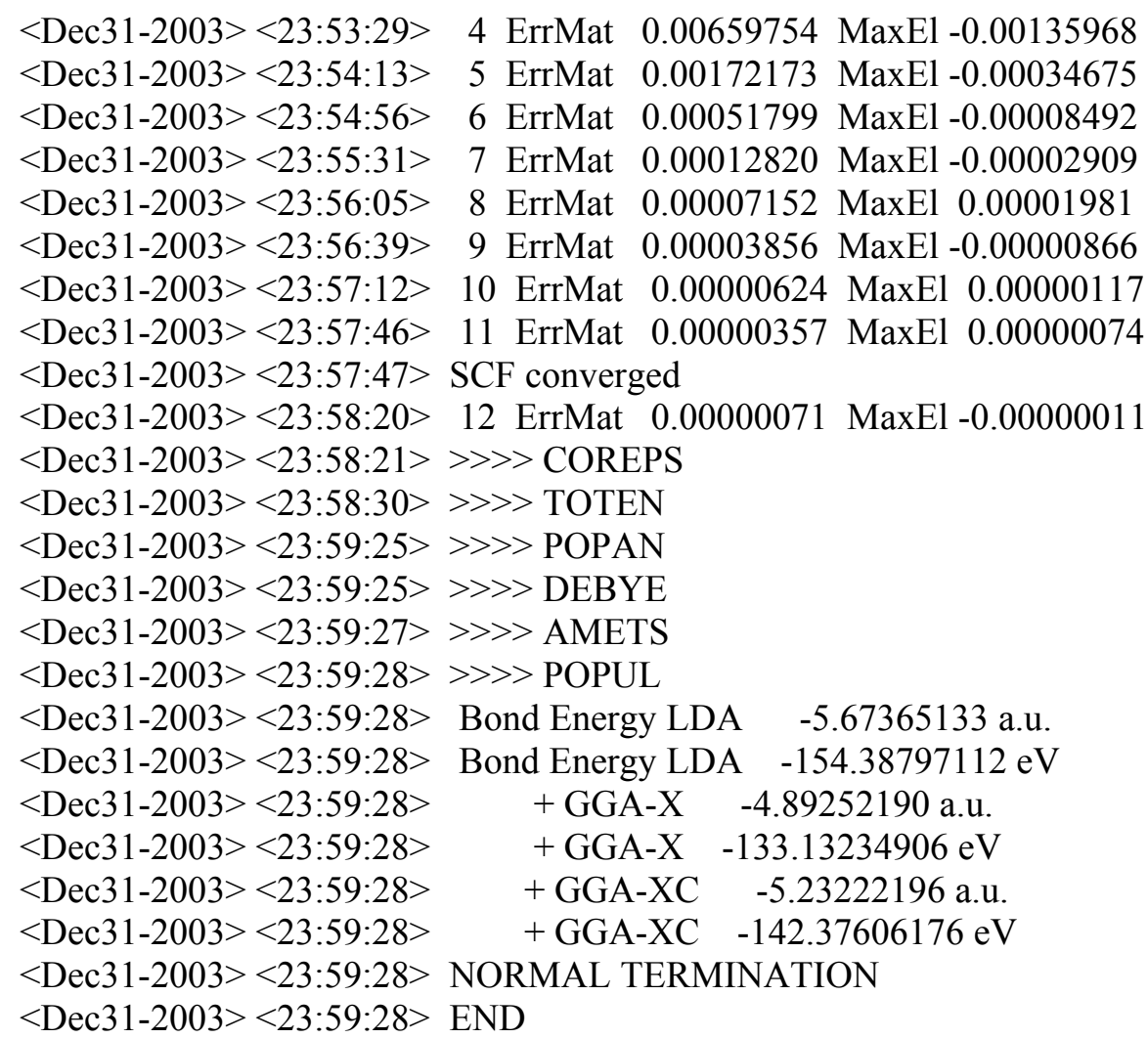

Selected Calculation Output for $\left\{\left[\mathrm{H}_{5} \mathrm{C}_{3} \mathrm{~N}_{2}\right] \mathrm{Cu}\right\}_{2}\left(\mu-\mathrm{CH}_{2}\right)\left(7-\mathrm{C}_{2}\right)$

\section{SYMMETRY C(2)}

RESTART restart21

MAXMEMORYUSAGE 330

REALMEMBLOCK 10

INTEGERMEMBLOCK 5

LOGICALMEMBLOCK 1

STRINGMEMBLOCK 15

$\mathrm{XC}$

GGA Becke Perdew

END

\section{ALLOW RELOPTINCONS}

\begin{tabular}{|c|c|c|}
\hline \multicolumn{3}{|c|}{ ATOMS } \\
\hline $\mathrm{C}$ & 0.000000 & -0.102917 \\
\hline $\mathrm{H}$ & $\begin{array}{lll}0.60 & 0.600000 & 0\end{array}$ & 0.599826 \\
\hline & -0.600000 & 0.599826 \\
\hline & $\begin{array}{lll}-1.267 & 0 & -1.505\end{array}$ & \\
\hline $\mathrm{N}$ & $\begin{array}{lll}-2.954 & 0.923 & -1.727\end{array}$ & \\
\hline
\end{tabular}




\begin{tabular}{llll}
$\mathrm{N}$ & -1.452 & -1.103 & -3.085 \\
$\mathrm{C}$ & -3.769 & 0.740 & -2.756 \\
$\mathrm{C}$ & -2.465 & -1.017 & -3.935 \\
$\mathrm{C}$ & -3.571 & -0.164 & -3.809 \\
$\mathrm{H}$ & -3.276 & 1.643 & -1.083 \\
$\mathrm{H}$ & -4.682 & 1.350 & -2.801 \\
$\mathrm{H}$ & -4.325 & -0.198 & -4.592 \\
$\mathrm{H}$ & -2.446 & -1.665 & -4.822 \\
$\mathrm{H}$ & -0.742 & -1.773 & -3.373 \\
$\mathrm{Cu}$ & 1.267 & 0 & -1.505 \\
$\mathrm{~N}$ & 2.954 & -0.923 & -1.727 \\
$\mathrm{~N}$ & 1.452 & 1.103 & -3.085 \\
$\mathrm{C}$ & 3.769 & -0.740 & -2.756 \\
$\mathrm{C}$ & 2.465 & 1.017 & -3.935 \\
$\mathrm{C}$ & 3.571 & 0.164 & -3.809 \\
$\mathrm{H}$ & 3.276 & -1.643 & -1.083 \\
$\mathrm{H}$ & 4.682 & -1.350 & -2.801 \\
$\mathrm{H}$ & 4.325 & 0.198 & -4.592 \\
$\mathrm{H}$ & 2.446 & 1.665 & -4.822 \\
$\mathrm{H}$ & 0.742 & 1.773 & -3.373 \\
$\mathrm{END}$ & & \multicolumn{2}{l}{}
\end{tabular}

Fragments

$\mathrm{H}$ t21.H

$\mathrm{N}$ t21.N

$\mathrm{Cu}$ t21.Cu

C t21.C

End

Relativistic scalar ZORA

CorePotentials t12.rel \&

$\mathrm{Cu} 1$

C 2

H 3

N 4

END

\section{GEOMETRY}

End

\section{END INPUT}

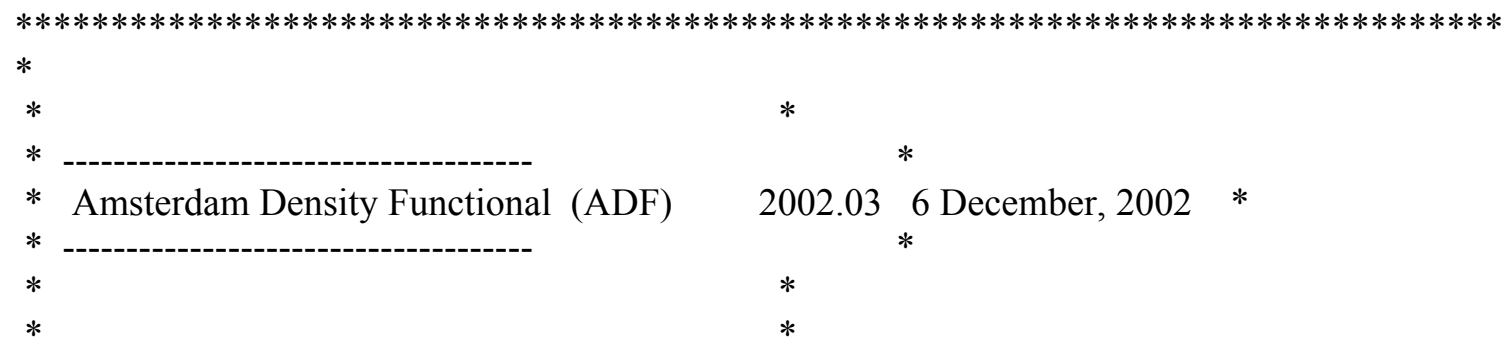




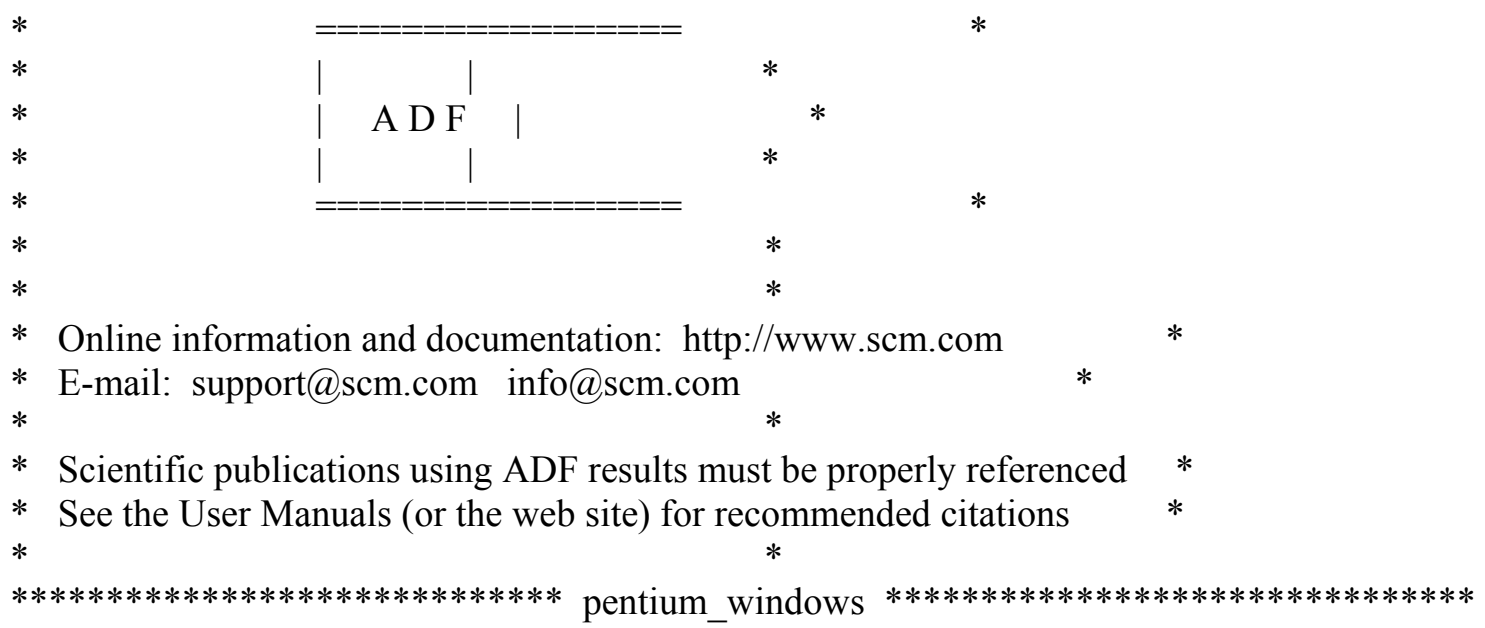

ADF 2002.03 RunTime: Mar14-2004 06:36:24 model $\mathrm{Cu} 2 \mathrm{CH} 2$

\section{A T T A C HED FILES}

Restart:

file : restart 21

jobid: ADF 2002.03 RunTime: Mar13-2004 18:13:52

title: model $\mathrm{Cu} 2 \mathrm{CH} 2$

Core Potentials:

file : t12.rel

MODEL PARAMETER S
DENSITY FUNCTIONAL POTENTIAL (scf)
LDA:
VWN
Gradient Corrections: Becke88 Perdew86
$==$ Not Default $==$
SPIN (restricted / unrestr.)
Molecule:
Restricted
Fragments:
Restricted
OTHER ASPECTS
Relativistic Corrections: scalar (ZORA,FrozenCore)
$* * * \operatorname{SPECIAL} * * *$
Core Treatment:
Frozen Orbital(s)
Electric Field: 
Hyperfine or Zeeman Interaction: ---

Fragment File(s)

$\mathrm{C}$ :

file : t21.C

jobid: ADF 2002.03 RunTime: Dec31-2003 23:01:27

title: Carbon (V, 1s frozen)

$\mathrm{H}$ :

file : $\mathrm{t} 21 . \mathrm{H}$

jobid: ADF 2002.03 RunTime: Dec31-2003 23:00:18

title: Hydrogen (V)

$\mathrm{Cu}$ :

file : $\mathrm{t} 21 . \mathrm{Cu}$

jobid: ADF 2002.03 RunTime: Dec31-2003 22:59:20

title: Copper (TZ2P+, $2 p$ frozen)

$\mathrm{N}$ :

file : $\mathrm{t} 21 . \mathrm{N}$

jobid: ADF 2002.03 RunTime: Dec31-2003 23:02:35

title: Nitrogen (V, 1s frozen)

**************************************************

* R U N T Y P E : GEOMETRY OPTIMIZATION *

$* * * * * * * * * * * * * * * * * * * * * * * * * * * * * * * * * * * * * * * * * * * * *$

$<$ Mar14-2004 $><07: 01: 48>$ E-test: old,new $=0.00000,-5.27040$ hartree

$<$ Mar14-2004 $><07: 01: 48>$ max gradient: 0.00148677 au/angstrom,radian

$<$ Mar14-2004 $><07: 01: 48>$ max cart. step: 0.00947121 angstrom

$<$ Mar14-2004 $><07: 01: 48>$ Geometry Converged

Coordinates in Geometry Cycle 2

\begin{tabular}{|c|c|c|c|}
\hline Atom & $X$ & Z & (Angstrom) \\
\hline 1.C & 0.000000 & 0.000000 & -0.198972 \\
\hline 2.H & 0.215271 & 0.868630 & 0.446742 \\
\hline. $\mathrm{H}$ & -0.215271 & -0.868630 & 0.446742 \\
\hline 4. $\mathrm{Cu}$ & -1.160498 & 0.019595 & -1.692578 \\
\hline $5 . \mathrm{N}$ & -2.937747 & 0.589571 & -1.317773 \\
\hline . $\mathrm{N}$ & -1.551636 & -0.635934 & -3.458678 \\
\hline 7.C & -3.953605 & 0.470496 & -2.165492 \\
\hline 8.C & -2.752377 & -0.661657 & -4.012631 \\
\hline. $\mathrm{C}$ & -3.910095 & -0.123423 & -3.432792 \\
\hline . $\mathrm{H}$ & -3.193283 & 1.035884 & 0512 \\
\hline I.H & -4.923736 & 0.872405 & -1.845619 \\
\hline 12.H & -4.834202 & -0.172621 & -4.003076 \\
\hline 13.H & -2.858459 & -1.132552 & -4.998928 \\
\hline 14.H & -0.827080 & -1.071924 & -4.025444 \\
\hline 15. Cu & 1.160498 & -0.019595 & -1.692578 \\
\hline $16 . \mathrm{N}$ & 2.937747 & -0.589571 & -1.317773 \\
\hline 17.N & 1.551636 & 0.635934 & -3.458678 \\
\hline 18.C & 3.953605 & -0.470496 & -2.165492 \\
\hline 19.C & 2.752377 & 0.661657 & -4.012631 \\
\hline 20.C & 3.910095 & 0.123423 & -3.432792 \\
\hline
\end{tabular}




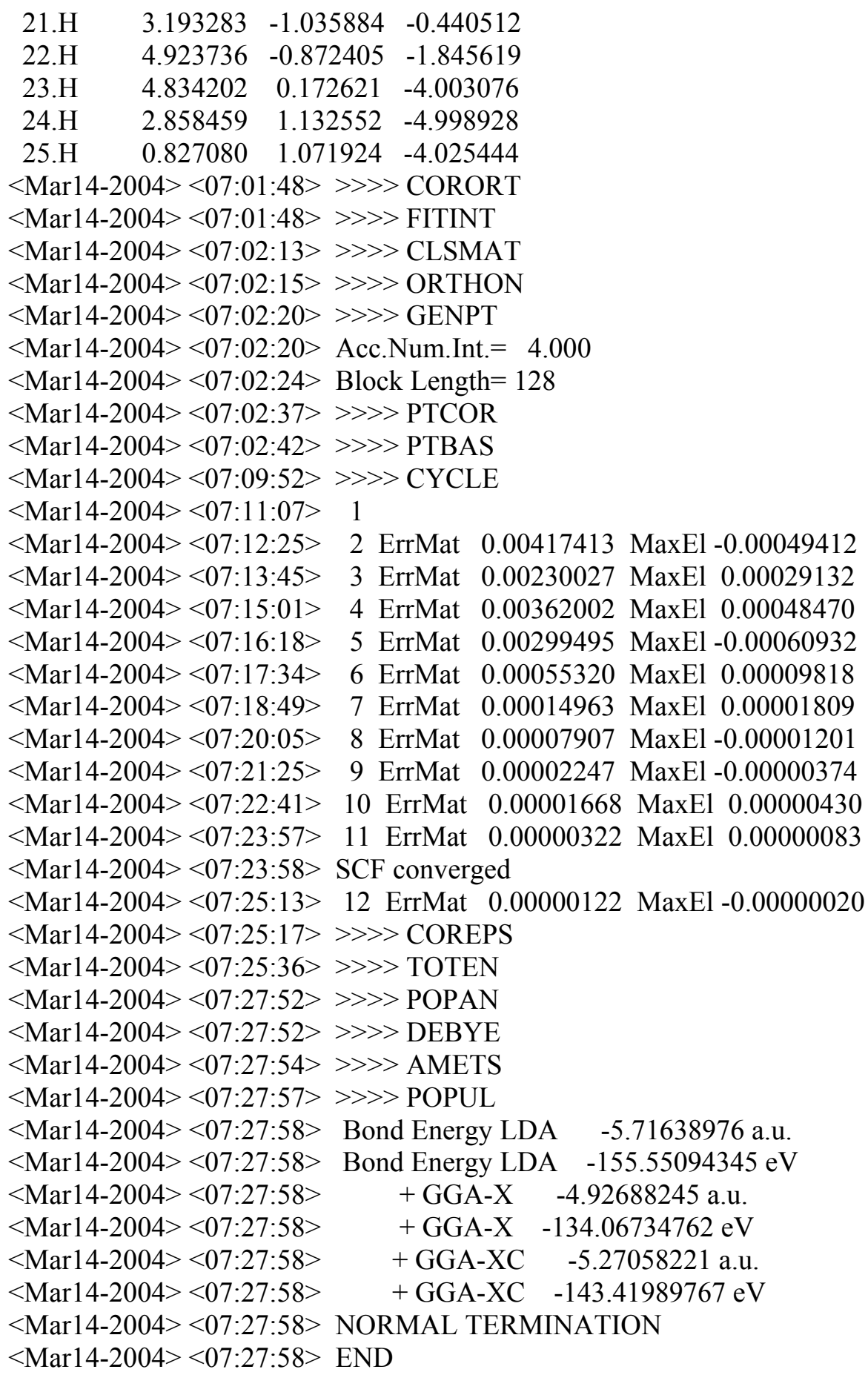

